\title{
A GROUPOID APPROACH TO PSEUDODIFFERENTIAL CALCULI
}

\author{
ERIK VAN ERP AND ROBERT YUNCKEN
}

\begin{abstract}
AвstRAct. We give an algebraic/geometric characterization of the classical pseudodifferential operators on a smooth manifold in terms of the tangent groupoid and its natural $\mathbb{R}_{+}^{\times}$-action. Specifically, a properly supported semiregular distribution on $M \times M$ is the Schwartz kernel of a classical pseudodifferential operator if and only if it extends to a smooth family of distributions on the range fibres of the tangent groupoid which is homogeneous for the $\mathbb{R}_{+}^{\times}$-action modulo smooth functions. Moreover, we show that the basic properties of pseudodifferential operators can be proven directly from this characterization. Further, with the appropriate generalization of the tangent bundle, the same definition applies without change to define pseudodifferential calculi on arbitrary filtered manifolds, in particular the Heisenberg calculus.
\end{abstract}

\section{CONTEnTs}

1. Introduction 2

1.1. Outline of the strategy 3

1.2. Structure of the paper 6

1.3. Acknowledgements 6

2. Convolution of distributions on Lie groupoids 6

2.1. Lie groupoids 6

2.2. Fibred distributions 7

2.3. Convolution of fibred distributions on a Lie groupoid 8

2.4. The ideal of smooth vertical densities 8

2.5. Proper distributions on a Lie groupoid 9

2.6. Proper Schwartz kernels 9

3. The tangent groupoid of a filtered manifold 10

3.1. Filtered manifolds 10

3.2. The osculating groupoid $T_{H} M$

3.3. Dilations 11

2010 Mathematics Subject Classification. Primary: 58J40; Secondary: 35S05, 47G30, 58H05, 22A22.

Key words and phrases. Pseudodifferential operators; Lie groupoids; filtered manifolds; hypoelliptic operators.

R. Yuncken was supported by the project SINGSTAR of the Agence Nationale de la Recherche, ANR-14-CE25-0012-01. 
3.4. The H-tangent groupoid 11

3.5. Global exponential coordinates 12

4. Pseudodifferential operators 13

4.1. The zoom action on the tangent groupoid 13

4.2. Definition of pseudodifferential operators 13

4.3. Cocvcles 14

4.4. Pseudolocality 14

4.5. Examples 15

5. Local exponential coordinates 16

5.1. Exponential cut-offs 16

5.2. Notation for graded vector spaces 16

5.3. Local exponential coordinates 17

6. Principal cosymbols 17

7. Svmbols 20

7.1. Fourier transforms on vector bundles 20

7.2. Full symbols 20

7.3. Homogeneity of the Fourier transform 21

8. Conormality and regularity 23

8.1. Svmbol estimates 23

8.2. Conormality 24

8.3. Algebra structure $\quad 25$

8.4. Regularity 25

9. Asvmptotic expansions 26

10. H-Ellipticity 28

11. Differential Operators 30

11.1. The universal enveloping algebra of a Lie algebroid 30

11.2. Principal svmbols 30

11.3. Differential operators as $H$-pseudodifferential operators 31

12. Classical Pseudodifferential Operators 32

Appendix A. 33

References $\quad 36$

\section{INTRODUCTION}

For the purposes of this article, a pseudodifferential calculus means simply an enlargement of the graded algebra of differential operators with two key properties. Firstly, the algebra should contain parametrices for some desired class of 'generalized elliptic' operators. Secondly, the pseudodifferential operators should be pseudolocal, that is, their Schwartz kernels should be $C^{\infty}$ off the diagonal. It is a simple exercise, for instance, to deduce hypoellipticity of the generalized elliptic operators from these two abstract properties. 
Defining classes of pseudodifferential operators can be a highly technical matter. In this article we propose a simple geometric/ algebraic definition of pseudodifferential operators which recovers the classical pseudodifferential operators, but which also applies without change to define the Heisenberg calculus ( $c f$. [BG88, Tay]) and its generalizations to other filtered manifolds (cf. Cum89, CGGP92, Mel82, Pon08]).

The method we describe is based upon the tangent groupoid. The tangent groupoid was used by Connes [Con94] as a geometric device for glueing a pseudodifferential operator to its principal symbol. We flip this around by showing that classical pseudodifferential operators can be completely characterized using the tangent groupoid. In this approach, the smoothness of the tangent groupoid accounts for the full asymptotic expansion of the symbol of a pseudodifferential operator, not just its principal term.

Furthermore, we will show that one can prove the basic properties of pseudodifferential operators directly from this geometric characterization. This allows us to define more general pseudodifferential calculi without needing to prescribe symbol or kernel classes explicitly.

1.1. Outline of the strategy. Let $P: C^{\infty}(M) \rightarrow C^{\infty}(M)$ be a differential operator of order $m$, given in local coordinates by

$$
P=\sum_{|\alpha| \leq m} a_{\alpha} \partial^{\alpha}
$$

Its highest order part at each point $x \in M$,

$$
P_{x}=\sum_{\alpha=m} a_{\alpha}(x) \partial^{\alpha},
$$

can be viewed as a smooth family of constant coefficient operators $P_{x}$ on the tangent spaces $T_{x} M$. The family $\left\{P_{x}\right\}_{x \in M}$ is the principal 'cosymbol' of $P$, i.e. the inverse Fourier transform of the principal symbol of $P$. The operator $P$ and its cosymbol $\left\{P_{x}\right\}_{x \in M}$ can be smoothly assembled into a single differential operator $\mathbb{P}$ on the tangent groupoid $\mathbb{T} M$.

Algebraically, $\mathbb{T} M$ is the disjoint union of the abelian groups $T_{x} M \cong \mathbb{R}^{n}$ and a one parameter family of pair groupoids $M \times M$ :

$$
\mathbb{T} M=(T M \times\{0\}) \sqcup\left(M \times M \times \mathbb{R}^{\times}\right) .
$$

The non-trivial aspect of the tangent groupoid is its smooth structure.

Let $\mathbb{P}$ be the differential operator on $\mathbb{T} M$ that restricts to $P_{x}$ on $T_{x} M$ and to $t^{m} P$ on each $M \times M \times\{t\}$ with $t \neq 0$, acting on the second factor in $M \times M$. The smooth structure on $\mathbb{T} M$ is precisely 
such that the coefficients of $\mathbb{P}$ are $C^{\infty}$ functions on $\mathbb{T} M$. Thus $\mathbb{P}$ is a left-invariant differential operator on $\mathbb{T} M$ that glues the operator $P$ (at $t=1$ ) to its principal cosymbol (at $t=0$ ).

Let us reformulate this in terms of distributional kernels on the groupoid $\mathbb{T} M$. Since each $P_{x}$ is a translation invariant operator on the abelian group $T_{x} M$, there exists a distribution $u_{x}$ on $T_{x} M$ such that $P_{x}$ acts as convolution with $u_{x}$,

$$
P_{x} \varphi=u_{x} * \varphi \quad u_{x}=P_{x}^{t} \delta_{0} \in \mathcal{E}^{\prime}\left(T_{x} M\right) \text {. }
$$

Likewise, $P$ has a Schwartz kernel $k$, which is smooth in the first variable, meaning that the function

$$
k: x \mapsto k_{x}=P^{t} \delta_{x} \in \mathcal{E}^{\prime}(M)
$$

is a smooth map from $M$ to $\mathcal{E}^{\prime}(M)$. If we now define

$$
\begin{array}{lll}
\mathbb{P}_{(x, 0)}=u_{x} \in \mathcal{E}^{\prime}\left(T_{x} M\right), & & t=0 \\
\mathbb{P}_{(x, t)}=t^{m} k_{x} \in \mathcal{E}^{\prime}(M), & & t \neq 0
\end{array}
$$

\{eq:diff_op_intro\}

then $\left(\mathbb{P}_{(x, t)}\right)_{(x, t) \in M \times \mathbb{R}}$ is a smooth family of compactly supported distributions in the fibres of the range map $r: \mathbb{T} M \rightarrow \mathbb{T} M^{(0)}=$ $M \times \mathbb{R}$.

The space of such smooth families of distributions on the $r$-fibres is denoted $\mathcal{E}_{r}^{\prime}(\mathbb{T} M)$; see Section 2 or [LMV15]. In analogy with the situation for Lie groups, these distributions form a convolution algebra in which the smooth functions-or more accurately, the smooth densities along the $r$-fibres-sit as a right ideal.

We need one more piece of structure. The tangent groupoid has a one parameter family of Lie groupoid automorphisms

$$
\alpha: \mathbb{R}_{+}^{\times} \mapsto \operatorname{Aut}(\mathbb{T} M)
$$

\{eq:classical_zoom\}

defined by

$$
\begin{aligned}
& \alpha_{\lambda}(x, y, t)=\left(x, y, \lambda^{-1} t\right) \quad(x, y, t) \in M \times M \times \mathbb{R}^{\times}, \\
& \alpha_{\lambda}(x, \xi, 0)=\left(x, \delta_{\lambda} \xi, 0\right) \quad \xi \in T_{x} M,
\end{aligned}
$$

where $\delta_{\lambda}$ denotes dilation of tangent vectors, $\delta_{\lambda} \xi=\lambda \xi$. Note that the smooth family of distributions $\mathbb{P}$ is homogeneous of weight $m$ for the action $\alpha$. In fact, the differential operators are characterized by this property.

Proposition 1. A semiregular kernel $P \in \mathcal{E}_{r}^{\prime}(M \times M)$ is the Schwartz \{prop:DOs\} kernel of a differential operator of order $m$ if and only if $P=\left.\mathbb{P}\right|_{t=1}$ for some $\mathbb{P} \in \mathcal{E}_{r}^{\prime}(\mathbb{T} M)$ such that $\alpha_{\lambda *} \mathbb{P}=\lambda^{m} \mathbb{P}$ for all $\lambda \in \mathbb{R}_{+}^{\times}$.

The motivating observation of this paper is that pseudodifferential operators admit a similar characterization. 
Theorem 2. A semiregular kernel $P \in \mathcal{E}_{r}^{\prime}(M \times M)$ is the Schwartz kernel $\{$ thm:PsiDOs\} of a properly supported classical pseudodifferential operator of order $m$ if and only if $P=\left.\mathbb{P}\right|_{t=1}$ for some $\mathbb{P} \in \mathcal{E}_{r}^{\prime}(\mathbb{T} M)$ such that $\alpha_{\lambda *} \mathbb{P}-\lambda^{m} \mathbb{P}$ is a smooth density for all $\lambda \in \mathbb{R}_{+}^{\times}$.

Now, if we replace $\mathbb{T} M$ by the appropriate tangent groupoid $\mathbb{T}_{H} M$ for a contact manifold [VE05, Pon06], or a more general filtered manifold [CP15, $\mathrm{vEY}]$, then the same definition can be used to produce versions of the Heisenberg calculus [BG88, Tay] or Melin's calculus [Mel82]. We will prove that the basic properties of pseudodifferential operators follow directly from our geometric definition.

To cite an instance of a need for such exotic pseudodifferential calculi, we point to Julg's proof of the Baum-Connes conjecture for $\operatorname{Sp}(n, 1)$ [Jul02], which requires a quaternionic analogue of the Heisenberg calculus. Our goal is to streamline such constructions. We want to demonstrate that, once an appropriate tangent groupoid has been constructed, the details of the corresponding pseudodifferential calculus follow automatically.

In particular, we prove, in the general setting of filtered manifolds, that our pseudodifferential operators form a $\mathbb{Z}$-filtered algebra $\Psi_{H}^{\bullet}(M)$; that restriction to $t=0$ in $\mathcal{E}_{r}^{\prime}\left(\mathbb{T}_{H} M\right)$ assigns a principal cosymbol to an operator in the calculus; that there is a short exact sequence

$$
0 \rightarrow \Psi_{H}^{m-1}(M) \rightarrow \Psi_{H}^{m}(M) \stackrel{\sigma_{m}}{\longrightarrow} \Sigma_{H}^{m}(M) \rightarrow 0 ;
$$

\{eq: short_exact_seq

where $\Sigma_{H}^{m}(M)$ is the (noncommutative) algebra of principal cosymbols; that the intersection $\cap_{m \in \mathbb{Z}} \Psi^{m}(M)$ is the ideal of properly supported smoothing operators $\Psi^{-\infty}(M)$; that $\Psi_{H}^{\bullet}(M)$ is complete with respect to asymptotic expansions; and that if an operator in $\Psi_{H}^{m}(M)$ has invertible principal cosymbol, then it has a parametrix in $\Psi_{H}^{-m}(M)$.

As an application, we immediately obtain the hypoellipticity of 'elliptic' elements in our calculi, where ellipticity here is taken in the abstract sense of having an invertible principal cosymbol. Moreover, given that there is a tangent groupoid associated to these calculi, Connes tangent groupoid method [Con94] should yield abstract index theorems for such operators, as is the case for subelliptic operators on a contact manifold [vE10].

Remark 3. A different geometric approach to the Heisenberg calculus is that of Epstein and Melrose [EM] using compactifications of the contangent bundle. This approach is currently limited to the standard calculus and the Heisenberg calculus, but it has other notable advantages, for instance an explicit noncommutative product on the symbol algebra and even a fusion of the standard and 
Heisenberg calculi. For comments on the relationship between the two, see Section 7.

1.2. Structure of the paper. In Section 2 we briefly recap convolution algebras of distributions on Lie groupoids. Section 3 recaps the tangent groupoid of a filtered manifold.

The main new results are contained in Sections 4-10, where we define the pseudodifferential operators and prove their fundamental properties. In Section 11 we discuss the case of differential operators. Finally, in Section 12 we prove that our construction recovers the classical pseudodifferential operators in the case of the classical tangent groupoid.

1.3. Acknowledgements. This article was inspired by an observation of Debord and Skandalis in their paper [DS14] which provides the first abstract characterization of the classical pseudodifferential operators in terms of the $\mathbb{R}_{+}^{\times}$-action on the tangent groupoid. We wish to thank them for many discussions, particularly during the time that the first author was Professor Invité at the Universite Blaise Pascal, Clermont-Ferrand II. Sincere thanks also go to Jean-Marie Lescure and Nigel Higson.

\section{Convolution of Distributions ON Lie groupoids}

The relevant framework for our approach to pseudodifferential operators is the convolution algebra of $r$-fibred distributions on a Lie groupoid. This generalizes both the algebra of Schwartz kernels of operators on $C^{\infty}(M)$ and the convolution algebra of compactly supported distributions on a Lie group. For a full discussion we refer to the recent article [LMV15] (the authors use the term distributions transverse to the range fibration, building on [AS11]). Here we only summarize the main points.

2.1. Lie groupoids. For the basic theory of Lie groupoids, we refer \{sec:notation\} to [Mac87] or [MM03].

Let $G$ be a Lie groupoid. We write $G^{(0)}$ for its unit space; $r, s$ : $G \rightarrow G^{(0)}$ for the range and source maps; $G^{x}=r^{-1}(\{x\})$ and $G_{x}=$ $s^{-1}(\{x\})$ for the range and source fibres at $x \in G^{(0)} ; G^{(2)}=G_{s} \times{ }_{r}$ $G \subseteq G \times G$ for the space of composable pairs; $m: G^{(2)} \rightarrow G$ for the product. The Lie algebroid, for us, will be the tangent bundle to the range fibres on the base, $\mathfrak{A} G=\left.(\operatorname{ker} d r)\right|_{G(0)}$, with Lie bracket $[\cdot, \cdot]$ on smooth sections $\Gamma(\mathfrak{A G})$ and anchor $\rho=d s: \mathfrak{A} G \rightarrow T G^{(0)}$.

Essentially, we will only need two examples, and combinations thereof. 
Example 4. The pair groupoid of a smooth manifold $M$ is $G=M \times$ $M \rightrightarrows G^{(0)}=M$ with range and source maps $r(x, y)=x, s(x, y)=y$ and composition $(x, y)(y, z)=(x, z)$. Its Lie algebroid is TM with the usual bracket of vector fields and the identity map as anchor.

Example 5. A smooth bundle of Lie groups is a groupoid $G \rightrightarrows G^{(0)}=$ $M$ with $r=s$. The fibres $G_{x}=G^{x}$ are Lie groups, and the Lie algebroid is a smooth bundle of Lie algebras with the pointwise Lie bracket and zero map as anchor. In particular, a vector bundle is a groupoid when viewed as a smooth bundle of abelian groups.

As already mentioned, the pair groupoid is the carrier of Schwartz kernels of pseudodifferential operators. Principal cosymbols are carried by the tangent bundle, which is a groupoid as in Example 5. For calculi on filtered manifolds, the tangent bundle is replaced by a smooth bundle of nilpotent groups called the osculating groupoid; see Section 3. The pair and osculating groupoids will be combined to form the tangent groupoid.

2.2. Fibred distributions. Let $\pi: E \rightarrow M$ be a submersion of $C^{\infty}$ $\{$ sec:fibered_distr manifolds. A subset $X \subset E$ is called $\pi$-proper if the restriction of $\pi$ to $X$ is a proper map.

Definition 6. A $\pi$-fibred distribution on $E$ is a continuous $C^{\infty}(M)$ linear map

$$
u: C^{\infty}(E) \rightarrow C^{\infty}(M),
$$

where the $C^{\infty}(M)$-module structure on $C^{\infty}(E)$ is induced by the pullback $\pi^{*}: C^{\infty}(M) \rightarrow C^{\infty}(E)$. We write $\langle u, f\rangle$ for the image of $f \in C^{\infty}(G)$ under $u$. The set of $\pi$-fibred distributions on $E$ is denoted $\mathcal{E}_{\pi}^{\prime}(E)$

Remark 7. More accurately, elemends $u \in \mathcal{E}_{\pi}^{\prime}(E)$ should be called $\pi$-properly supported $\pi$-fibred distributions, since the continuity of $u$ with respect to the Frechet topology on $C^{\infty}(E)$ forces $u$ to be supported in a $\pi$-proper set.

A $\pi$-fibred distribution $u$ can equivalently be given by a smooth family $\left(u_{x}\right)_{x \in M}$ of distributions on the fibres $u_{x} \in \mathcal{E}_{\pi}^{\prime}\left(\pi^{-1}\{x\}\right)$ such that the support $\bigcup_{x \in M} \operatorname{supp}\left(u_{x}\right)$ is $\pi$-proper. Smoothness here means the following: if $M_{0} \times F \hookrightarrow E$ is any local fibration chart, with $M_{0} \subseteq M$ and $F \subseteq \mathbb{R}^{k}$ open sets, then restricting the family $\left(u_{x}\right)_{x \in M_{0}}$ to $M_{0} \times F$ yields an element of $C^{\infty}\left(M_{0} ; \mathcal{E}^{\prime}(F)\right)$. The correspondence between the two pictures is given by

$$
\langle u, f\rangle(x)=\left\langle u_{x}, f\right\rangle, \quad f \in C^{\infty}(E) .
$$


2.3. Convolution of fibred distributions on a Lie groupoid. Now we specialize to the case of a Lie groupoid $G \rightrightarrows M$. If $u \in \mathcal{E}_{r}^{\prime}(G)$ is an $r$-fibred distribution, we will write $\left(u^{x}\right)_{x \in M}$ for the associated smooth family of distributions on the $r$-fibres $G^{x}$. Likewise, $\left(v_{x}\right)_{x \in M}$ denotes the smooth family of distributions on the $s$-fibres associated to $v \in \mathcal{E}_{S}^{\prime}(G)$.

The convolution $u * v$ of two $r$-fibred distributions $u, v \in \mathcal{E}_{r}^{\prime}(G)$ is defined by

$\langle u * v, \varphi\rangle(x)=\left\langle u^{x}(\gamma),\left\langle v^{s(\gamma)}(\beta), \varphi(\gamma \beta)\right\rangle\right\rangle, \quad \varphi \in C^{\infty}(G), x \in G^{(0)}$

(here $\gamma \in G^{x}$ and $\beta \in G^{s(\gamma)}$ are placeholder variables). Likewise, convolution of $s$-fibred distributions $u, v \in \mathcal{E}_{s}^{\prime}(G)$ is given by

$\langle u * v, \varphi\rangle(x)=\left\langle v_{x}(\gamma),\left\langle u_{r(\gamma)}(\beta), \varphi(\beta \gamma)\right\rangle\right\rangle, \quad \varphi \in C^{\infty}(G), x \in G^{(0)}$.

Example 8. Let $G=M \times M$ be the pair groupoid of a manifold $M$. Then

$$
\mathcal{E}_{r}^{\prime}(M \times M) \cong C^{\infty}\left(M ; \mathcal{E}^{\prime}(M)\right) \cong C^{\infty}(M) \hat{\otimes} \mathcal{E}^{\prime}(M),
$$

i.e., distributions which are semiregular in the first variable. These are precisely the Schwartz kernels of continuous linear operators $C^{\infty}(M) \rightarrow C^{\infty}(M)$; see [Trè80, Ch.51]. Convolution in $\mathcal{E}_{r}^{\prime}(M \times M)$ corresponds to the usual composition law for Schwartz kernels:

$$
u * v(x, z)=\int_{y \in M} u(x, y) v(y, z) .
$$

\subsection{The ideal of smooth vertical densities.}

Definition 9. A subset $X$ of a groupoid $G$ is proper if it is both $r$ and s-proper, i.e. both $r: X \rightarrow M$ and $s: X \rightarrow M$ are proper maps.

Properness is a common support condition. For instance, the classical notion of proper support for a Schwartz kernel $p \in \mathcal{D}^{\prime}(M \times M)$ corresponds to proper support on the pair groupoid.

We denote by $\Omega_{r}$ the bundle of densities tangent to the range fibres of $G$. The space of properly supported smooth sections of $\Omega_{r}$ will be denoted $C_{\mathrm{p}}^{\infty}\left(G ; \Omega_{r}\right)$.

There is a canonical inclusion $C_{\mathrm{p}}^{\infty}\left(G ; \Omega_{r}\right) \hookrightarrow \mathcal{E}_{r}^{\prime}(G)$ given by integration along the range fibres:

$$
\langle f, \varphi\rangle(x)=\int_{\gamma \in G^{x}} \varphi(\gamma) f(\gamma), \quad f \in C_{\mathrm{p}}^{\infty}\left(G ; \Omega_{r}\right), \varphi \in C^{\infty}(G) .
$$

Likewise, there is a canonical inclusion $C_{p}^{\infty}\left(G ; \Omega_{S}\right) \hookrightarrow \mathcal{E}_{S}^{\prime}(G)$ where $\Omega_{S}$ is the bundle of densities tangent to the s-fibres. \{sec:convolution\} 
Proposition 10 ([LMV15]). $C_{\mathrm{p}}^{\infty}\left(G, \Omega_{r}\right)$ is a right ideal in $\mathcal{E}_{r}^{\prime}(G)$ and $C_{\mathrm{p}}^{\infty}\left(G, \Omega_{s}\right)$ is a left ideal in $\mathcal{E}_{\mathcal{S}}^{\prime}(G)$.

Example 11. On the pair groupoid (Example 4$), C_{\mathrm{p}}^{\infty}\left(M \times M ; \Omega_{r}\right)$ consists of the Schwartz kernels of properly supported smoothing operators. Note that any $u \in \mathcal{E}_{r}^{\prime}(M \times M)$ is smooth in the first variable while $v \in C_{\mathrm{p}}^{\infty}\left(M \times M ; \Omega_{r}\right)$ is smooth in both variables. Therefore $u * v$ will also be smooth in both variables. The same cannot be expected for $v * u$.

2.5. Proper distributions on a Lie groupoid. In order to have the smooth densities as a two-sided ideal, one must restrict to the intersection of $\mathcal{E}_{r}^{\prime}(G)$ and $\mathcal{E}_{s}^{\prime}(G)$. This requires a little attention.

A transverse measure on a Lie groupoid $G$ is a positive density $\mu$ on the object space $G^{(0)}$. Fixing a transverse measure allows us to integrate $r$ - and $s$-fibred distributions to global distributions on $G$, via the maps

$$
\begin{aligned}
\mu_{r}: \mathcal{E}_{r}^{\prime}(G) \rightarrow \mathbb{D}^{\prime}(G) ; & u \mapsto \mu \circ u=\int_{G^{(0)}}\langle u, \cdot\rangle(x) d \mu(x), \\
\mu_{s}: \mathcal{E}_{s}^{\prime}(G) \rightarrow \mathbb{D}^{\prime}(G) ; & u \mapsto \mu \circ u=\int_{G^{(0)}}\langle u, \cdot\rangle(x) d \mu(x) .
\end{aligned}
$$

Definition 12. The set of proper distributions on $G$, denoted $\mathcal{D}_{\mathrm{p}}^{\prime}(G)$, is the intersection

$$
\mathcal{D}_{\mathrm{p}}^{\prime}(G)=\mu_{r}\left(\mathcal{E}_{r}^{\prime}(G)\right) \cap \mu_{s}\left(\mathcal{E}_{s}^{\prime}(G)\right) \quad \subset \mathcal{D}^{\prime}(G) .
$$

An $r$-fibred distribution $u \in \mathcal{E}_{r}^{\prime}(G)$ or an s-fibred distribution $v \in$ $\mathcal{E}_{s}^{\prime}(G)$ is called proper if $\mu_{r}(u)$ or $\mu_{s}(v)$ is proper, respectively. We denote the set of proper $r$-fibred distributions as $\mathcal{E}_{r, s}^{\prime}(G)$, and the set of proper $s$-fibred distributions as $\mathcal{E}_{s, r}^{\prime}(G)$.

In other words, a properly supported distribution $u \in \mathcal{D}^{\prime}(G)$ is proper if it can be disintegrated along the $r$-fibres as well as along the $s$-fibres (in the language of [AS11, LMV15] it is "transversal to both $r$ and $\left.s^{\prime \prime}\right)$. The definition of properness is independent of the choice of transverse measure $\mu$, although the specific disintegrations into $r$ - and $s$-fibred distributions will depend on $\mu$. As algebras, $\mathcal{E}_{r, s}^{\prime}(G)$ and $\mathcal{E}_{s, r}^{\prime}(G)$ are isomorphic, via the map $\mu_{s}^{-1} \circ \mu_{r}$.

2.6. Proper Schwartz kernels. We finish this section with a charac$\{$ sec:pair_groupoid\} terization of proper distributions on the pair groupoid $G=M \times M$ in terms of Schwartz kernels. For convenience, we fix once and for all a transverse measure $\mu$ which will allow us to identify smooth functions and smooth densities on $M$ via $f \mapsto f \mu$. It also gives us a 
bilinear form on $C_{\mathrm{c}}^{\infty}(M)$ :

$$
\langle f, g\rangle=\int_{M} f(x) g(x) d \mu(x), \quad f, g \in C_{c}^{\infty}(M) .
$$

The discussion in [Trè80, Ch.51] yields the following.

Proposition 13. Let $P: C_{\mathrm{c}}^{\infty}(M) \rightarrow \mathcal{D}^{\prime}(M)$ be a continuous linear oper\{prop:regular\} ator with Schwartz kernel $p$. The following are equivalent:

(1) $P$ maps $C_{\mathrm{C}}^{\infty}(M)$ to itself and admits a transpose $P^{t}: C_{\mathrm{C}}^{\infty}(M) \rightarrow$ $C_{\mathrm{c}}^{\infty}(M)$ such that $\langle f, P g\rangle=\left\langle P^{t} f, g\right\rangle$ for any $f, g \in C_{\mathrm{c}}^{\infty}(M)$,

(2) P extends to continuous operators $C_{\mathrm{c}}^{\infty}(M) \rightarrow C_{\mathrm{c}}^{\infty}(M), C^{\infty}(M) \rightarrow$ $C^{\infty}(M), \mathcal{E}^{\prime}(M) \rightarrow \mathcal{E}^{\prime}(M)$ and $\mathcal{D}^{\prime}(M) \rightarrow \mathcal{D}^{\prime}(M)$.

(3) $p$ is a proper distribution on the pair groupoid.

(4) $p$ is properly supported and semiregular in both variables.

Remark 14. The Schwartz kernel of $P^{t}$ is $p^{t}=\left(\mu_{s}^{-1} \circ \mu_{r}\right)(p) \circ \iota^{*}$ where $\iota:(x, y) \mapsto(y, x)$ is the inverse on $M \times M$.

Proposition 13 shows that pseudodifferential operators in any reasonable calculus (on a manifold without boundary) should have Schwartz kernels in $\mathcal{E}_{r, s}^{\prime}(M \times M)$.

\section{THE TANGENT GROUPOID OF A FILTERED MANIFOLD}

We will work in the generality of filtered manifolds to demonstrate the versatility of our approach. The reader who is only interested in the classical pseudodifferential calculus, may choose to substitute this section with an account of the classical tangent groupoid, e.g. [Con94].

A construction of the tangent groupoid of a filtered manifold has been described in a recent preprint of Choi and Ponge [CP15] (they use the name Carnot manifold). See also [Con94, vE05, Pon06, JvE10] for historical antecedents. We will need an intrinsic (i.e. coordinatefree) construction of the tangent groupoid which we have described separately in the paper [vEY]. Here we give a quick description to fix notation.

\subsection{Filtered manifolds.}

Definition 15. ([Mel82]) A filtered manifold is a smooth manifold $M$ equipped with a filtration of the tangent bundle TM by vector bundles $M \times\{0\}=H^{0} \leq H^{1} \leq \cdots \leq H^{N}=T M$ such that $\Gamma\left(H^{\bullet}\right)$ is a Lie algebra filtration, i.e.

$$
\left[\Gamma\left(H^{i}\right), \Gamma\left(H^{j}\right)\right] \subseteq \Gamma\left(H^{i+j}\right) .
$$

Here we are using the convention that $H^{i}=T M$ for $i>N$.

Sections $X \in \Gamma\left(H^{i}\right)$ will be referred to as vector fields of $H$-order $\leq i$, written $\operatorname{ord}_{H}(X) \leq i$. 
Example 16. (1) Any manifold $M$ can be equipped with the trivial filtration of depth one: $H^{1}=T M$. This is the relevant filtration for describing the classical pseudodifferential operators in terms of the standard tangent groupoid.

(2) Contact manifolds, and more generally Heisenberg manifolds, are filtered manifolds of depth 2. See [FS74, BG88].

(3) Parabolic geometries are examples of filtered manifolds which are modelled locally on the flag variety of a semisimple Lie group; see [ČS09].

3.2. The osculating groupoid $T_{H} M$. Let $M$ be a filtered manifold. We write $\mathfrak{t}_{H} M$ for the associated graded bundle, i.e.,

$$
\mathfrak{t}_{H} M=\bigoplus_{i=1}^{n} \mathfrak{t}_{H}^{i} M, \quad \text { where } \mathfrak{t}_{H}^{i} M:=H^{i} / H^{i-1} .
$$

We denote the grading maps by $\sigma_{i}: H^{i} \rightarrow \mathfrak{t}_{H}^{i} M$. (This notation is chosen to coincide with the principal cosymbol maps; see Section 11.) For any $X \in \Gamma\left(H^{i}\right), Y \in \Gamma\left(H^{j}\right)$, and $f, g \in \Gamma(M)$ we have

$$
[f X, g Y] \equiv f g[X, Y] \bmod \Gamma\left(H^{i+j-1}\right),
$$

\{eq:Lie_bracket\}

which implies that the Lie bracket is a pointwise operation on $\mathfrak{t}_{H} M$. In other words, $\mathfrak{t}_{H} M$ is a smooth bundle of graded nilpotent Lie algebras over $M$. Equipped with the zero anchor, $\mathfrak{t}_{H} M$ is thus a Lie algebroid.

Definition 17. The osculating groupoid $T_{H} M$ is the smooth bundle \{def :osculating gro of connected, simply connected nilpotent Lie groups obtained by exponentiating the Lie algebroid $\mathfrak{t}_{H} M$.

Explicitly, $T_{H} M=\mathfrak{t}_{H} M$ as a smooth fibre bundle, and each fibre is equipped with the group law given by the Baker-CampbellHausdorff formula. Thus $T_{H} M$ is a Lie groupoid, with range and source maps both equal to the bundle projection.

3.3. Dilations. Any graded vector bundle $E=\bigoplus_{i=1}^{N} E_{i}$ is equipped \{sec:dilations\} with a canonical family of dilations $\left(\delta_{\lambda}\right)_{\lambda \in \mathbb{R}}$, which are bundle endomorphisms such that $\delta_{\lambda}$ acts on $E_{i}$ by multiplication by $\lambda^{i}$.

In the case of the Lie algebroid $\mathfrak{t}_{H} M=\bigoplus_{i=1}^{N} \mathfrak{t}_{H}^{i} M$, the dilations $\delta_{\lambda}$ are Lie algebroid endomorphisms. Therefore, they are also groupoid endomorphisms of $T_{H} M$ (i.e. fibrewise group endomorphisms).

3.4. The $H$-tangent groupoid. Algebraically, the $H$-tangent group$\{$ sec:H-tangent_grou oid is the disjoint union of the osculating groupoid $T_{H} M$ with a family of pair groupoids indexed by $\mathbb{R} \backslash\{0\}$ :

$$
\mathbb{T}_{H} M=T_{H} M \times\{0\} \sqcup(M \times M) \times \mathbb{R}^{\times}
$$

\{eq:H-tangent_group 
This is to be understood as a smooth family of Lie groupoids over $\mathbb{R}$, in that the allowable compositions are

$$
\begin{aligned}
(z, y, t)(y, x, t)=(z, x, t), & x, y, z \in M, t \in \mathbb{R}^{\times}, \\
(x, \xi, 0)(x, \eta, 0)=(x, \xi \eta, 0), & x \in M, \xi, \eta \in T_{H} M_{x} .
\end{aligned}
$$

The component $(M \times M) \times \mathbb{R}^{\times}$has the obvious smooth structure. In order to glue in the groupoid $T_{H} M$ at $t=0$, we blow up the diagonal of $M \times M$ by the dilations $\delta_{t^{-1}}$, as we now describe.

Definition 18. A degrading of $\mathfrak{t}_{H} M$ is an isomorphism of vector bundles $\psi: \mathfrak{t}_{H} M \rightarrow T M$ such that the restriction of $\psi$ to $\mathfrak{t}_{H}^{i} M$ is right inverse to the grading map $\sigma_{i}$ for each $i$.

Let $\nabla$ be a graded connection on the bundle $\mathfrak{t}_{H} M$ over $M$, i.e., $\nabla$ restricts to a connection on each $\mathfrak{t}_{H}^{i} M$. We can transport this to a connection $\nabla^{\psi}$ on TM by fixing a degrading $\psi$ :

$$
\nabla_{X}^{\psi} Y=\psi\left(\nabla_{X}\left(\psi^{-1} Y\right)\right), \quad X, Y \in \Gamma(T M) .
$$

We then use the associated geometric exponential

$$
\exp _{x}^{\nabla^{\psi}}: T_{x} M \rightarrow M
$$

to define the groupoid exponential $]^{1}$

$$
\begin{aligned}
\operatorname{Exp}^{\psi, \nabla}: \mathfrak{t}_{H} M \times \mathbb{R} & \longrightarrow T_{H} M \times\{0\} \sqcup M \times M \times \mathbb{R}^{\times} ; \\
(x, v, t) & \longmapsto \begin{cases}\left(x, \exp _{x}^{\nabla^{\psi}}\left(\psi\left(\delta_{t} v\right)\right), t\right) & \text { if } t \neq 0, \\
(x, v, 0) & \text { if } t=0,\end{cases}
\end{aligned}
$$

(6) $\{$ eq:EExp\}

The smooth structure on $\mathbb{T}_{H} M$ is defined by declaring that this be a smooth map. Let us be more precise.

\subsection{Global exponential coordinates.}

Definition 19. A proper open neighbourhood $U \subseteq T M$ of the zero $\{$ sec:global_exponen $\{$ def : domain_of_inje section will be called a domain of injectivity (for the connection $\nabla$ and degrading $\psi$ ) if the exponential map

$$
\operatorname{Exp}: U \rightarrow M \times M ; \quad(x, v) \mapsto\left(x, \exp _{x}^{\nabla^{\psi}} v\right)
$$

\{eq:domain_of_injec

is injective.

If $U$ is a domain of injectivity, then the groupoid exponential $\mathbb{E x p}^{\psi, \nabla}$ of Equation (6) is a diffeomorphism from the open set

$$
\left\{(x, v, t) \in \mathfrak{t}_{H} M \times \mathbb{R} \mid\left(x, \psi\left(\delta_{t} v\right)\right) \in U\right\},
$$

(8) \{eq:dom_inj_2\}

\footnotetext{
${ }^{1}$ We recall that, for us, the Lie algebroid is the tangent space to the range fibres, so Equation (6) represents the exponential along the range fibres.
} 
to its image

$$
\mathbb{U}:=\left(T_{H} M \times\{0\}\right) \sqcup\left(\operatorname{Exp}^{\nabla^{\psi}}(U) \times \mathbb{R}^{\times}\right) \quad \subset \quad \mathbb{T}_{H} M . \quad \text { (9) } \quad \text { eq: exponential_pat }
$$

Such a set $\mathbb{U}$ will be called a global exponential coordinate patch for $\mathbb{T}_{H} M$. It is an open neighbourhood of both the osculating groupoid $T_{H} M \times\{0\}$ and the object space $\mathbb{T}_{H} M^{(0)}=M \times \mathbb{R}$.

\section{Pseudodifferential operators}

$\{\sec : \operatorname{Rsth\theta \theta }\}$

4.1. The zoom action on the tangent groupoid. The tangent groupoid comes with equipped with an action of $\mathbb{R}_{+}^{\times}$. It was of central importance in [DS14]. We are taking the liberty of giving it a pithy name.

Definition 20. The zoom action $\alpha: \mathbb{R}_{+}^{\times} \rightarrow \operatorname{Aut}\left(\mathbb{T}_{H} M\right)$ is the smooth \{def : zoom\} one-parameter family of Lie groupoid automorphisms defined as follows:

$$
\begin{array}{ll}
\alpha_{\lambda}(x, y, t)=\left(x, y, \lambda^{-1} t\right) & \text { if }(x, y) \in M \times M, t \neq 0, \\
\alpha_{\lambda}(x, \xi, 0)=\left(x, \delta_{\lambda}(\xi), 0\right) & \text { if }(x, \xi) \in T_{H} M, t=0 .
\end{array}
$$

\{eq:zoom $\}$

To confirm that the zoom action is smooth, we rewrite it in exponential coordinates. Specifically, we have the smooth action $\tilde{\alpha}$ of $\mathbb{R}_{+}^{\times}$ on $\mathfrak{t}_{H} M \times \mathbb{R}$ defined by

$$
\tilde{\alpha}_{\lambda}(x, \xi, t)=\left(x, \delta_{\lambda}(\xi), \lambda^{-1} t\right),
$$

\{eq:zoom_in_coords $\}$

which satisfies $\alpha_{\lambda} \circ \mathbb{E x p} \psi, \nabla=\mathbb{E x p} \psi, \nabla \circ \tilde{\alpha}_{\lambda}$.

The zoom action induces a one-parameter family of automorphisms $\left(\alpha_{\lambda *}\right)_{\lambda \in \mathbb{R}_{+}^{\times}}$on the convolution algebra $\mathcal{E}_{r}^{\prime}\left(\mathbb{T}_{H} M\right)$. Specifically, for $u \in \mathcal{E}_{r}^{\prime}\left(\mathbb{T}_{H} M\right), f \in C^{\infty}\left(\mathbb{T}_{H} M\right)$, we have

$$
\left\langle\alpha_{\lambda *} u, f\right\rangle(x, t)=\left\langle u, \alpha_{\lambda}^{*} f\right\rangle(x, \lambda t) \quad \text { for }(x, t) \in M \times \mathbb{R} .
$$

The action preserves the subalgebra $\mathcal{E}_{r, s}^{\prime}\left(\mathbb{T}_{H} M\right)$ of proper $r$-fibred distributions, as well as the ideal of properly supported smooth densities.

4.2. Definition of pseudodifferential operators. We can now give our definition of pseudodifferential operators.

Definition 21. A properly supported $r$-fibred distribution $\mathbb{P} \in \mathcal{E}_{r}^{\prime}\left(\mathbb{T}_{H} M\right)^{\{d e f}$ is called essentially homogeneous of weight $m \in \mathbb{R}$ if

$$
\alpha_{\lambda *} \mathbb{P}-\lambda^{m} \mathbb{P} \in C_{\mathrm{p}}^{\infty}\left(\mathbb{T}_{H} M ; \Omega_{r}\right) \quad \text { for all } \lambda \in \mathbb{R}_{+}^{\times} .
$$

\{eq:essentially_hom

The space of such distributions will be denoted $\rrbracket_{H}^{m}(M)$. 
For $t \in \mathbb{R}$, we will denote by $\mathbb{P}_{t}$ the restriction of $\mathbb{P}$ to the fibre $\left.\mathbb{T}_{H} M\right|_{t}$, so

$$
\begin{aligned}
& \mathbb{P}_{t} \in \mathcal{E}_{r}^{\prime}(M \times M) \quad \text { for } t \neq 0, \\
& \mathbb{P}_{0} \in \mathcal{E}_{r}^{\prime}\left(T_{H} M\right) .
\end{aligned}
$$

Definition 22. A Schwartz kernel $P \in \mathcal{E}_{r}^{\prime}(M \times M)$ will be called $\{$ def:PsiDOs\} an H-pseudodifferential kernel of order $m$ (or, by abuse of terminology, an $H$-pseudodifferential operator of order $m$ ) if $P=\mathbb{P}_{1}$ for some $\mathbb{P} \in \mathbb{v}_{H}^{m}(M)$. In this case, we say that $\mathbb{P}$ extends $P$. The set of $H$ pseudodifferential kernels of order $m$ will be denoted $\Psi_{H}^{m}(M)$.

To be clear, the $H$-pseudodifferential operator associated to the kernel $P \in \Psi_{H}^{m}(M)$ is given by

$$
\mathrm{Op}(P): C^{\infty}(M) \rightarrow C^{\infty}(M) ; \quad(\mathrm{Op}(P) f)(x)=\int_{y \in M} P(x, y) f(y) .
$$

However, we will generally blur the distinction between $P$ and the associated operator $\mathrm{Op}(P)$.

4.3. Cocycles. The smooth differences (12) will appear frequently in the ensuing analysis, so we shall give them a name.

Definition 23. The function $F: \mathbb{R}_{+}^{\times} \rightarrow C_{\mathrm{p}}^{\infty}\left(\mathbb{T}_{H} M ; \Omega_{r}\right)$ defined by

$$
F: \lambda \mapsto F_{\lambda}:=\lambda^{-m} \alpha_{\lambda *} \mathbb{P}-\mathbb{P}
$$

$\{$ eq: cocycle_def $\}$

will be called the cocycle associated to $\mathbb{P} \in \Downarrow_{H}^{m}(M)$.

These cocycles satisfy the cocycle condition

$$
F_{\lambda_{1} \lambda_{2}}=\lambda_{1}^{-m} \alpha_{\lambda_{1} *} F_{\lambda_{2}}+F_{\lambda_{1}}
$$

\{eq: cocycle\}

4.4. Pseudolocality. Our definition of $\Downarrow_{H}^{m}(M)$ forces the singular support of an $H$-pseudodifferential kernel to lie on the diagonal.

Proposition 24. Every $\mathbb{P} \in \Downarrow_{H}^{m}(M)$ is smooth off the unit space, in the \{prop:smooth_off_th sense that the restriction of $\mathbb{P}$ to $\mathbb{T}_{H} M \backslash \mathbb{T}_{H} M^{(0)}$ is equal to a smooth density. Hence every $P \in \Psi_{H}^{m}(M)$ is smooth off the diagonal.

Proof. The singular support of $\mathbb{P} \in \Downarrow_{H}^{m}(M)$ is invariant under the zoom action. If $\gamma \in \mathbb{T}_{H} M \backslash \mathbb{T}_{H} M^{(0)}$ then its orbit under the zoom action is not proper. Since $\mathbb{P}$ has proper support by definition, the result follows.

Remark 25. In Section 8.2, we will see that the definition also forces $H$-pseudodifferential kernels to be proper fibred distributions, so they satisfy the equivalent conditions of Proposition 13. That will require a bit more effort. 


\subsection{Examples.}

Example 26. Let $M$ be trivially filtered. If $P$ is (the Schwartz kernel $\{e x:$ diff_op\} of) a differential operator of order $m$, the discussion in the introduction shows that it extends to a smooth family of $r$-fibred distributions on $\mathbb{T} M$,

$$
\mathbb{P}_{t}= \begin{cases}t^{m} P, & t \neq 0, \\ \sigma_{m}(P), & t=0,\end{cases}
$$

where $\sigma_{m}(P)=\left(P_{x}\right)_{x \in M}$ is the principal part. The resulting $r$ fibred distribution $\mathbb{P}$ is homogeneous on the nose, in the sense that $\alpha_{\lambda *} \mathbb{P}-\lambda^{m} \mathbb{P}=0$ for all $\lambda \in \mathbb{R}_{+}^{\times}$. Thus differential operators are $H$-pseudodifferential operators, according to our definition.

This example generalizes easily to differential operators on filtered manifolds once the appropriate notion of order is defined. We defer this to Section 11 .

In particular, on any filtered manifold, the identity operator $I$ : $C^{\infty}(M) \rightarrow C^{\infty}(M)$ is an $H$-pseudodifferential operator of order 0 . An extension $\mathbb{I} \in \mathbb{\Psi}_{H}^{0}(M)$ is the $r$-fibred distribution $\mathbb{I} \in \mathcal{E}_{r}^{\prime}\left(\mathbb{T}_{H} M\right)$ given by restriction to the unit space: $(\mathbb{I}, f)=\left.f\right|_{M \times \mathbb{R}}$.

Example 27. Let $M=\mathbb{R}^{n}$ with the trivial filtration: $H^{1}=T M$. With $\{$ ex:logarithmic_sin the standard connection, the exponential map is a global diffeomorphism

$$
\begin{aligned}
\mathbb{E x p}: T M \times \mathbb{R} & \rightarrow \mathbb{T} M ; & & \\
(x, \xi, t) & \mapsto(x, x-t \xi, t), & & \text { if } t \neq 0, \\
(x, \xi, 0) & \mapsto(x, \xi, 0), & & \text { if } t=0 .
\end{aligned}
$$

Consider the fibred distribution $\mathbb{P} \in \mathcal{E}_{r}^{\prime}(M)$ whose pull-back $\widetilde{\mathbb{P}}:=$ $\mathbb{E x p} * \mathbb{P}$ via exponential coordinates is

$$
\widetilde{\mathbb{P}}(x, \xi, t)=\log |\xi| d \xi .
$$

In standard coordinates,

$$
\begin{array}{ll}
\mathbb{P}(x, y, t)=\log \left(|t|^{-1}|x-y|\right) d y, & \text { if } t \neq 0, \\
\mathbb{P}(x, \xi, t)=\log |\xi| d \xi, & \text { if } t \neq 0 .
\end{array}
$$

From Equation (11) we see that

$$
\tilde{\alpha}_{\lambda *} \widetilde{\mathbb{P}}(x, \xi, t)=\log \left|\lambda^{-1} \xi\right| d\left(\lambda^{-1} \xi\right)=\lambda^{-n}(\log |\xi|-\log |\lambda|) d \xi,
$$

so

$$
\left(\tilde{\alpha}_{\lambda *} \widetilde{\mathbb{P}}-\lambda^{-n} \widetilde{\mathbb{P}}\right)(x, \xi, t)=-\lambda^{-n} \log |\lambda| d \xi
$$

which is a smooth density for all $\lambda \in \mathbb{R}_{+}^{\times}$. Thus $\mathbb{P}$ is essentially homogeneous of weight $-n$. It is not properly supported, but one can easily resolve this by multiplying $\mathbb{P}$ by an exponential cut-off 
function (see the next section). Thus, after a properly supported cut-off, $\mathbb{P}_{1}(x, y)=\log |x-y| d y$ is an $H$-pseudodifferential kernel.

Remark 28. In some other kernel-based approaches to pseudodifferential calculi (e.g. [CGGP92]), logarithmic singularities need to be included specially. One of the advantages of the present approach is that they enter naturally. Compare also [Tay, Section I.2].

\section{LOCAL EXPONENTIAL COORDINATES}

A few preparatory details are in order before we proceed to the analysis of $H$-pseudodifferential operators. An important point to make is that the essential data of an $H$-pseudodifferential operator is carried on a neighbourhood of the unit space $\mathbb{T}_{H} M^{(0)}=M \times \mathbb{R}$ and the osculating groupoid $\left.\mathbb{T}_{H} M\right|_{0}=T_{H} M$ inside $\mathbb{T}_{H} M$.

In Section 3.5 we observed that global exponential coordinates give a linearization for this neighbourhood. We now add a few extra details.

5.1. Exponential cut-offs. Let $\varphi_{1} \in C^{\infty}(M \times M)$ be a smooth bump function which equals 1 on a neighbourhood of the diagonal and zero outside the image of a domain of injectivity $U$ as in Equation (7). The smooth function $\varphi$ on $\mathbb{T}_{H} M$ defined by

$$
\begin{cases}\varphi(x, y, t)=\varphi_{1}(x, y), & \text { if } t \neq 0, \\ \varphi(x, \xi, 0)=1 & \text { if } t=0 .\end{cases}
$$

will be called an exponential cut-off function. It is constant 1 on a neighbourhood of $\left.\mathbb{T}_{H} M^{(0)} \cup \mathbb{T}_{H} M\right|_{0}$, and 0 outside of the exponential coordinate patch $\mathbb{U}=\left(T_{H} M \times\{0\}\right) \sqcup\left(\operatorname{Exp}^{\nabla}(U) \times \mathbb{R}^{\times}\right)$. It is also invariant under the zoom action.

Any $\mathbb{P} \in \mathbb{\Psi}_{H}^{m}(M)$ differs from $\varphi \mathbb{P}$ by an element of $C_{\mathrm{p}}^{\infty}\left(\mathbb{T}_{H} M ; \Omega_{r}\right)$. Thus, we may routinely assume that $\mathbb{P}$ is supported in an exponential coordinate patch.

We will then write $\widetilde{\mathbb{P}} \in \mathcal{E}_{r}^{\prime}\left(\mathfrak{t}_{H} M \times \mathbb{R}\right)$ for the pullback of $\mathbb{P}$ under global exponential coordinates (6), conscious of the fact that this depends upon choices of degrading $\psi$, graded connection $\nabla$ and exponential cut-off.

5.2. Notation for graded vector spaces. The following terminology for graded vector spaces $V$ will be used throughout the paper.

A graded basis for $V$ is a basis $\left\{e_{1}, \ldots, e_{n}\right\}$ such that each element $e_{j}$ is contained in some $V_{i}$. The corresponding linear coordinates $\xi=$ $\left(\xi_{1}, \ldots, \xi_{n}\right)$ will be called a graded coordinate system. We will write $d_{j}=\operatorname{deg}\left(e_{j}\right)$. The homogeneous dimension of $V$ is $d_{H}=\sum_{j=1}^{n} d_{j}$. If $\mathbf{a}=$ $\left(a_{1}, \ldots, a_{n}\right) \in \mathbb{N}^{n}$ is a multi-index with respect to these coordinates, 
we write $|\mathbf{a}|=\sum_{j} a_{j}$ for its usual order and $|\mathbf{a}|_{H}=\sum_{j} a_{j} d_{j}$ for its homogeneous order.

5.3. Local exponential coordinates. Let $\mathbb{R}^{n} \supset M_{0} \hookrightarrow M$ be a co$\{$ sec: charts\} ordinate chart for $M$ with compact closure upon which all of the bundles $H^{i}$ are trivializable. We identify $M_{0}$ with its image in $M$.

We thus have a trivialization of graded vector bundles

$$
M_{0} \times\left. V \stackrel{\cong}{\longrightarrow} \mathfrak{t}_{H} M\right|_{M_{0}}
$$

where $V$ is a graded vector space. The map

$$
M_{0} \times V \times\left.\mathbb{R} \stackrel{\cong}{\longrightarrow} \mathfrak{t}_{H} M\right|_{M_{0}} \times \mathbb{R} \stackrel{\mathbb{E x p}^{\psi, \nabla}}{\longrightarrow} \mathbb{T}_{H} M,
$$

\{eq:trivialization\}

will be called a local exponential coordinate chart. We will reuse $r$ to denote the bundle projection

$$
r: M_{0} \times V \times \mathbb{R} \rightarrow M_{0} \times \mathbb{R}
$$

which corresponds to the range projection of $\mathbb{T}_{H} M$.

Now choose coordinates $x=\left(x_{i}\right)$ for $M_{0}$, graded coordinates $\xi=\left(\xi_{i}\right)$ for $V$, and let $t$ be the standard coordinate on $\mathbb{R}$. The system $(x, \xi, t)$ will be referred to as local exponential coordinates. By invoking a partition of unity on $M$, we may generally assume that an element $\mathbb{P} \in \Downarrow_{H}^{m}(M)$ is supported in the image of a local exponential coordinate chart, in which case we write $\widetilde{\mathbb{P}} \in \mathcal{E}_{r}^{\prime}\left(M_{0} \times V \times \mathbb{R}\right)$ for its pullback via local exponential coordinates.

\section{Principal cosymbols}

The following proposition and its corollary will allow us to unambiguously define the principal part of an $\mathrm{H}$-pseudodifferential operator.

Lemma 29. Let $\mathbb{P} \in \Downarrow_{H}^{m}(M)$. If $\mathbb{P}_{1} \in C_{\mathrm{p}}^{\infty}\left(M \times M ; \Omega_{r}\right)$ then $\mathbb{P}_{0} \in$ \{lem:smooth_cosymbo $C_{\mathrm{p}}^{\infty}\left(T_{H} M\right)$.

Proof. Essential homogeneity immediately implies that $\mathbb{P}_{t} \in C_{\mathrm{p}}^{\infty}(M \times$ $\left.M ; \Omega_{r}\right)$ for all $t \neq 0$. We use an Arzela-Ascoli argument to show that the limiting distribution $\mathbb{P}_{0}$ is also smooth.

We work with the pullback $\widetilde{\mathbb{P}} \in \mathcal{E}_{r}^{\prime}\left(M_{0} \times V \times \mathbb{R}\right)$ of $\mathbb{P}$ via local exponential coordinates. Let $\omega=\left(\omega_{x, t}\right)_{(x, t) \in M_{0} \times \mathbb{R}}$ denote the constant family of Lebesgue measures on $V$ so that $\left.\widetilde{\mathbb{P}}\right|_{M_{0} \times V \times \mathbb{R}^{\times}}=p \omega$ for some $p \in C^{\infty}\left(M_{0} \times V \times \mathbb{R}^{\times}\right)$. Likewise, we write the cocycle associated to $\widetilde{\mathbb{P}}$ as $F_{\lambda}=f_{\lambda} \omega$ where $f_{\lambda} \in C^{\infty}\left(M_{0} \times V \times \mathbb{R}\right)$. 
In exponential coordinates, the zoom action of Equation (11) acts on fibrewise Lebesgue measure by $\tilde{\alpha}_{\lambda *} \omega=\lambda^{-d_{H}} \omega$. It follows that

$$
f_{\lambda}=\lambda^{-m-d_{H}} \tilde{\alpha}_{\lambda^{-1}}^{*} p-p
$$

$\{$ eq:function_cocycl

Differentiating this gives

$\partial_{x}^{\mathbf{a}} \partial_{\tilde{\xi}}^{\mathbf{b}} f_{\lambda}(x, \xi, t)=\lambda^{-m-d_{H}-|\mathbf{b}|_{H}}\left(\partial_{x}^{\mathbf{a}} \partial_{\tilde{\xi}}^{\mathbf{b}} p\right)\left(x, \delta_{\lambda^{-1}}(\xi), \lambda t\right)-\left(\partial_{x}^{\mathbf{a}} \partial_{\tilde{\xi}}^{\mathbf{b}} p\right)(x, \xi, t)$

for any multi-indices $\mathbf{a}, \mathbf{b} \in \mathbb{N}^{n}$. By fixing $\lambda=\frac{1}{2}$, we get the following estimate in uniform norms for every $t \in[0,1]$ :

$$
\begin{aligned}
\left|2^{m+d_{H}+|\mathbf{b}|_{H}}\left\|\left.\partial_{x}^{\mathbf{a}} \partial_{\xi}^{\mathbf{b}} p\right|_{\frac{1}{2} t}\right\|_{\overline{M_{0}} \times V \times\left\{\frac{1}{2} t\right\}}-\left\|\left.\partial_{x}^{\mathbf{a}} \partial_{\xi}^{\mathbf{b}} p\right|_{t}\right\| \|_{\overline{M_{0}} \times V \times\{t\}}\right| \\
\leq\left\|\partial_{x}^{\mathbf{a}} \partial_{\tilde{\zeta}}^{\mathbf{b}} f_{\frac{1}{2}}\right\|_{\overline{M_{0}} \times V \times[0,1]},
\end{aligned}
$$

where $\left.p\right|_{t}$ denotes the restriction of $p$ to $M \times V \times\{t\}$.

Let us put $m^{\prime}=m+d_{H}+|\mathbf{b}|_{H}$. We obtain

$$
\begin{aligned}
& \left\|\left.\partial_{x}^{\mathbf{a}} \partial_{\tilde{\zeta}}^{\mathbf{b}} p\right|_{2^{-k}}\right\| \overline{M_{0}} \times V \\
& =\left(\left\|\left.\partial_{x}^{\mathbf{a}} \partial_{\xi}^{\mathbf{b}} p\right|_{2^{-k}}\right\|_{\overline{M_{0}} \times V}-\left.2^{-m^{\prime}}\left\|\left.\partial_{x}^{\mathbf{a}} \partial_{\xi}^{\mathbf{b}} p\right|_{2^{-(k-1)}}\right\|\right|_{\overline{M_{0}} \times V}\right) \\
& +\left(\left.2^{-m^{\prime}}\left\|\left.\partial_{x}^{\mathbf{a}} \partial_{\tilde{\xi}}^{\mathbf{b}} p\right|_{2^{-(k-1)}}\right\|\left\|_{\overline{M_{0}} \times V}-2^{-2 m^{\prime}}\right\| \partial_{x}^{\mathbf{a}} \partial_{\tilde{\xi}}^{\mathbf{b}} p\right|_{2^{-(k-2)}} \|_{\overline{M_{0}} \times V}\right) \\
& +\cdots+\left(2^{-(k-1) m^{\prime}}\left\|\left.\partial_{x}^{\mathbf{a}} \partial_{\tilde{\xi}}^{\mathbf{b}} p\right|_{2^{-1}}\right\|_{\overline{M_{0}} \times V}-2^{-k m^{\prime}}\left\|\left.\partial_{x}^{\mathbf{a}} \partial_{\xi}^{\mathbf{b}} p\right|_{1}\right\|_{\overline{M_{0}} \times V}\right) \\
& +2^{-k m^{\prime}}\left\|\left.\partial_{x}^{\mathbf{a}} \partial_{\tilde{\zeta}}^{\mathbf{b}} p\right|_{1}\right\|_{\overline{M_{0}} \times V} \\
& \leq\left.\left(\sum_{j=1}^{k} 2^{-j m^{\prime}}\right)\left\|\partial_{x}^{\mathbf{a}} \partial_{\xi}^{\mathbf{b}} f_{\frac{1}{2}}\right\|\left\|_{\overline{M_{0}} \times V \times[0,1]}+2^{-k m^{\prime}}\right\| \partial_{x}^{\mathbf{a}} \partial_{\xi}^{\mathbf{b}} p\right|_{1} \|_{\overline{M_{0}} \times V} .
\end{aligned}
$$

for every $k \in \mathbb{N}$. If $m^{\prime}>0$, this is bounded as $k \rightarrow \infty$. That is, for all multi-indices $\mathbf{a}, \mathbf{b}$ with $|\mathbf{b}|_{H}>-m-d_{H}$, the sequence of functions $\left(\left.p\right|_{2^{-k}}\right)_{k \in \mathbb{N}}$ is bounded with respect to the seminorm $\left\|\partial_{x}^{\mathbf{a}} \partial_{\xi}^{\mathbf{b}} \bullet\right\|_{\overline{M_{0}} \times V}$. Since $\operatorname{supp}(p) \cap\left(\overline{M_{0}} \times V \times[0,1]\right)$ is compact, the derivatives $\partial_{x}^{\mathbf{a}} \partial_{\tilde{\xi}}^{\mathbf{b}} p$ with $|\mathbf{b}|_{H} \leq-m-d$ are bounded in terms of those with $|\mathbf{b}|_{H}>$ $-m-d_{H}$. By the Arzela-Ascoli Theorem, it admits a limit point $\left.p\right|_{0} \in C^{\infty}\left(\overline{M_{0}} \times V\right)$. But $\widetilde{\mathbb{P}}_{2^{-k}} \rightarrow \widetilde{\mathbb{P}}_{0}$ as distributions, so $\widetilde{\mathbb{P}}_{0}=\left.p\right|_{0} \omega$.

Corollary 30. Let $\mathbb{P}, \mathbb{Q} \in \Downarrow_{H}^{m}(M)$. If $\mathbb{P}_{1} \equiv \mathbb{Q}_{1}$ modulo $C_{\mathrm{p}}^{\infty}\left(M \times M ; \Omega_{r}\right)$ then $\mathbb{P}_{0} \equiv \mathbb{Q}_{0}$ modulo $C_{\mathrm{p}}^{\infty}\left(T_{H} M ; \Omega_{r}\right)$.

Definition 31. For $m \in \mathbb{R}$, we define

$$
\begin{aligned}
\Sigma_{H}^{m}(M):=\left\{K \in \mathcal{E}_{r}^{\prime}\left(T_{H} M\right) / C_{\mathrm{p}}^{\infty}\left(T_{H} M ; \Omega_{r}\right) \mid\right. \\
\left.\delta_{\lambda_{*}} K=\lambda^{m} K \text { for all } \lambda \in \mathbb{R}_{+}^{\times}\right\} .
\end{aligned}
$$

Elements of $\Sigma_{H}^{m}(M)$ will be called cosymbols of order $m$ on $M$. 
We will not bother to use a separate notation for an essentially homogeneous $r$-fibred distribution $K \in \mathcal{E}_{r}^{\prime}\left(T_{H} M\right)$ and its class in $\Sigma_{H}^{m}(M)$. Of course, as a distribution, the homogeneity condition on $K$ is $\delta_{\lambda *} K-\lambda^{m} K \in C_{\mathrm{p}}^{\infty}\left(T_{H} M ; \Omega_{r}\right)$.

Definition 32. We define the cosymbol map

$$
\sigma_{m}: \Psi_{H}^{m}(M) \rightarrow \Sigma_{H}^{m}(M)
$$

as follows. For $P \in \Psi_{H}^{m}(M)$, choose any $\mathbb{P} \in \Psi_{H}^{m}(M)$ with $\mathbb{P}_{1}=P$ and set $\sigma_{m}(P):=\mathbb{P}_{0}\left(\right.$ modulo $C_{\mathrm{p}}^{\infty}\left(T_{H} M ; \Omega_{r}\right)$ ).

This is well-defined by Corollary 30 .

Lemma 33. The cosymbol map $\sigma_{m}$ is surjective.

Proof. Consider a cosymbol $K \in \mathcal{E}_{r}^{\prime}\left(T_{H} M\right)$ of order $m$. Define the constant family $\widetilde{\mathbb{K}} \in \mathcal{E}_{r}^{\prime}\left(\mathfrak{t}_{H} M \times \mathbb{R}\right)$ by $\widetilde{\mathbb{K}}_{t}=K$ for all $t \in \mathbb{R}$. Then $\widetilde{\mathbb{K}}$ is essentially homogeneous for the action $\left(\tilde{\alpha}_{\lambda}\right)$ of Equation (11). This can be transferred to an essentially homogeneous distribution $\mathbb{K}$ on $\mathbb{T}_{H} M$ by use of an exponential cut-off (Section 5.1). It has $\mathbb{K}_{0}=K$.

Proposition 34. Let $\mathbb{P} \in \Downarrow_{H}^{m}(M), k \in \mathbb{Z}$. The following are equivalent:

(1) $\mathbb{P}=t^{k} \mathbb{Q}$ for some $\mathbb{Q} \in \mathcal{E}_{r}^{\prime}\left(\mathbb{T}_{H} M\right)$,

(2) the pullback $\widetilde{\mathbb{P}} \in \mathcal{E}_{r}^{\prime}\left(\mathfrak{t}_{H} M \times \mathbb{R}\right)$ of $\mathbb{P}$ under any global exponential coordinates satisfies $\left.\left(\partial_{t}^{j} \mathbb{P}\right)\right|_{t=0}=0$ for $j=0, \ldots, k-1$.

In this case, $\mathbb{Q} \in \Psi_{H}^{m-k}(M)$.

Proof. Since $\widetilde{\mathbb{P}} \in \mathcal{E}_{r}^{\prime}\left(\mathfrak{t}_{H} M \times \mathbb{R}\right) \cong C^{\infty}\left(\mathbb{R}, \mathcal{E}_{r}^{\prime}\left(\mathfrak{t}_{H} M\right)\right)$, the equivalence of the vanishing conditions (1) and (2) is immediate. The cocycle $\widetilde{F}$ associated to $\widetilde{\mathbb{P}}$ also vanishes to order $k-1$ in $t$, so $F_{\lambda}=t^{k} G_{\lambda}$ for some $G_{\lambda} \in C_{\mathrm{p}}^{\infty}\left(\mathbb{T}_{H} M ; \Omega_{r}\right)$. For all $t \neq 0$ we have

$$
\begin{aligned}
\left(\lambda^{-m+k} \alpha_{\lambda *} \mathbb{Q}-\right. & \mathbb{Q})_{t}=\lambda^{-m+k} \mathbb{Q}_{\lambda t}-\mathbb{Q}_{t} \\
& =t^{-k}\left(\lambda^{-m} \mathbb{P}_{\lambda t}-\mathbb{P}_{t}\right)=t^{-k}\left(\lambda^{-m} \alpha_{\lambda *} \mathbb{P}-\mathbb{P}\right)_{t}=\left.G_{\lambda}\right|_{t}
\end{aligned}
$$

By continuity, we also get $\left(\lambda^{-m+k} \alpha_{\lambda *} \mathbb{Q}-\mathbb{Q}\right)_{0}=\left.G_{\lambda}\right|_{0}$. Thus $\mathbb{Q} \in$ $\mathbb{\Psi}_{H}^{m-k}(M)$.

Corollary 35. $\Psi_{H}^{m-1}(M)=\operatorname{ker} \sigma_{m} \subseteq \Psi_{H}^{m}(M)$ for every $m \in \mathbb{Z}$.

Proof. The map $\Downarrow_{H}^{m-1}(M) \hookrightarrow \mathbb{\Psi}_{H}^{m}(M) ; \mathbb{P} \mapsto t \mathbb{P}$ yields the inclusion $\Psi_{H}^{m-1}(M) \hookrightarrow \operatorname{ker} \sigma_{m}$. It is surjective by Proposition 34 .

This proves the short exact sequence (3) of the introduction. 
Although Fourier transforms-"symbols"-play no role in our definition of pseudodifferential operators, they are (unsurprisingly) necessary for proving the main analytic properties. We begin by recalling a few facts about vector bundle Fourier transforms.

7.1. Fourier transforms on vector bundles. Let $\pi: E \rightarrow M$ be a vector bundle and $\pi^{\prime}: E^{*} \rightarrow M$ its dual bundle.

Recall (see e.g. [CR08, DS14]) that there is a well-defined space $\mathcal{S}_{\pi}(E)$ of fibre-wise Schwartz class functions on $E$, with the following Fréchet seminorms:

$$
\|f\|_{K, \mathbf{a}, \mathbf{b}, \mathbf{c}}=\sup _{x \in K, \xi \in V}\left|\xi^{\mathbf{a}} \partial_{\tilde{\xi}}^{\mathbf{b}} \partial_{x}^{\mathbf{c}} f(x, \xi)\right|,
$$

where $(x, \xi) \in M_{0} \times V \hookrightarrow E$ are linear bundle coordinates, $K \subset M_{0}$ is a compact subset, and $\mathbf{a}, \mathbf{b}, \mathbf{c} \in \mathbb{N}^{n}$ are multi-indices. One can likewise define the Schwartz class vertical densities $\mathcal{S}_{\pi}\left(E ; \Omega_{\pi}\right)$.

The fibrewise Fourier transform $\mathcal{F}_{\pi}: \mathcal{S}_{\pi}\left(E ; \Omega_{\pi}\right) \rightarrow \mathcal{S}_{\pi^{\prime}}\left(E^{*}\right)$ is defined by $u \mapsto \hat{u}$ where

$$
\hat{u}(\eta)=\left(u_{x}, e^{-i(\eta, \cdot)}\right), \quad \eta \in E_{x}^{*}, x \in M,
$$

$\{$ eq :Schwarz_seminor

This extends to a map between the tempered fibred distributions $\mathcal{S}_{\pi}^{\prime}(E) \rightarrow \mathcal{S}_{\pi^{\prime}}^{\prime}\left(E^{*} ; \Omega_{\pi}^{*}\right)$, which are the $C^{\infty}(M)$-linear duals of the Schwartz spaces. In particular, $\mathcal{E}_{\pi}^{\prime}(E)$ is a subset of $\mathcal{S}_{\pi}^{\prime}(E)$. The Fourier transform maps $\mathcal{E}_{\pi}^{\prime}(E)$ into $C^{\infty}\left(E^{*}\right)$.

7.2. Full symbols. Let $P \in \mathcal{E}_{r}^{\prime}(M \times M)$ be an $H$-pseudodifferential kernel on $M$. After passing to exponential coordinates we could use the fibrewise Fourier transform to define the full symbol of $P$. In fact, more important is the Fourier transform of the essentially homogeneous distribution $\mathbb{P} \in \mathcal{E}_{r}^{\prime}\left(\mathbb{T}_{H} M\right)$ which extends it, since this is where we see the essential homogeneity.

Definition 36. Let $\mathbb{P} \in \Psi_{H}^{m}(M)$ and let $\widetilde{\mathbb{P}} \in \mathcal{E}_{r}^{\prime}\left(\mathfrak{t}_{H} M \times \mathbb{R}\right)$ be its pullback under some global exponential coordinates. We will write $\hat{\mathbb{P}} \in C^{\infty}\left(\mathfrak{t}_{H}^{*} M \times \mathbb{R}\right)$ for the Fourier transform of $\widetilde{\mathbb{P}}$. For brevity, we will refer to $\hat{\mathbb{P}}$ as the Fourier transform of $\mathbb{P}$, conscious once again that this depends upon the choice of global exponential coordinates and an exponential cut-off function.

The (full) symbol of an $H$-pseudodifferential operator $P=\mathbb{P}_{1}$ is defined as $\hat{P}=\hat{\mathbb{P}}_{1}$, with the same caveats.

It is understood that any statements we make regarding $\hat{\mathbb{P}}$ or $\hat{P}$ will hold regardless of the choice of exponential coordinates and cut-off.

$\{$ def:PPhat $\}$

$\{$ eq:fibrewise_Fouri

$\{$ sec:full_symbols\} 
Example 37. Consider the case of $M=\mathbb{R}^{n}$ with trivial filtration, so $\mathfrak{t}_{H} M=T M$. Using the standard connection $\nabla$, the exponential map is

$$
\begin{array}{rlrl}
\operatorname{Exp}^{\nabla}: \mathfrak{t}_{H} M \times \mathbb{R} & \rightarrow \mathbb{T}_{H} M & \\
(x, \xi, t) & \mapsto(x, x+t \xi, t), & & t \neq 0 \\
(x, \xi, 0) & \mapsto(x, \xi, 0), & & t=0 .
\end{array}
$$

Pulling back $P=\mathbb{P}_{1}$ by exponential coordinates gives $\widetilde{P}(x, \xi)=$ $P(x, x+\xi)$. Therefore the symbol $\hat{P}$ is defined by exactly the same formula which converts a classical pseudodifferential kernel to its symbol—see Equation (18.1.7) of [Hör85].

7.3. Homogeneity of the Fourier transform. Roughly speaking, the Fourier transform converts homogeneity of a distribution $u$ near zero into homogeneity of $\hat{u}$ near infinity. In this section, we give a precise application of this principal to $\Downarrow_{H}^{m}(M)$. This is perhaps the fundamental point in the analysis of our pseudodifferential operators.

Let $E=\bigoplus_{i} E_{i}$ be a graded vector bundle. The dual bundle $E^{*}$ is also graded, thanks to the canonical decomposition $E^{*} \cong \bigoplus_{i} E_{i}^{*}$. We denote by $\left(\delta_{\lambda}\right)$ and $\left(\delta_{\lambda}^{\prime}\right)$ the canonical families of dilations on $E$ and $E^{*}$, respectively (see Section 3.3). Note that the fibrewise Fourier transform $\mathcal{F}_{\pi}: \mathcal{E}_{\pi}^{\prime}(E) \rightarrow C^{\infty}\left(E^{*}\right)$ is compatible with the dilations:

$$
\widehat{\delta_{\lambda_{*}} u}=\delta_{\lambda}^{\prime *} \hat{u}, \quad \text { for all } u \in \mathcal{E}_{\pi}^{\prime}(E) \text {. }
$$

We apply this to $\mathbb{P} \in \mathbb{\Psi}_{H}^{m}(M)$. From Equation (11), the zoom action on $\widetilde{\mathbb{P}}$ in exponential coordinates is given by

$$
\left.\left(\tilde{\alpha}_{\lambda *} \widetilde{\mathbb{P}}\right)\right|_{t}=\delta_{\lambda *}\left(\widetilde{\mathbb{P}}_{\lambda t}\right), \quad t \in \mathbb{R}, \lambda \in \mathbb{R}^{\times} .
$$

Taking fibrewise Fourier transforms we get

$$
\begin{aligned}
\left(\widehat{\alpha_{\lambda *} \mathbb{P}}\right)(x, \eta, t)=\left(\delta_{\lambda *} \widetilde{\mathbb{P}}_{(x, \lambda t)}, e^{-i(\eta, \cdot)}\right) \\
=\left(\widetilde{\mathbb{P}}_{(x, \lambda t)}, e^{-i\left(\delta_{\lambda}^{\prime}(\eta), \cdot\right)}\right)=\hat{\mathbb{P}}\left(x, \delta_{\lambda}^{\prime} \eta, \lambda t\right),
\end{aligned}
$$

where $(x, \eta, t) \in \mathfrak{t}_{H}^{*} M \times \mathbb{R}$. That is,

$$
\widehat{\tilde{\alpha}_{\lambda *} \widetilde{\mathbb{P}}}=\beta_{\lambda}^{*} \hat{\mathbb{P}},
$$

where $\beta_{\lambda}: \mathfrak{t}_{H}^{*} M \times \mathbb{R} \rightarrow \mathfrak{t}_{H}^{*} M \times \mathbb{R}$ is defined by

$$
\beta_{\lambda}(x, \eta, t)=\left(x, \delta_{\lambda}^{\prime}(\eta), \lambda t\right) .
$$

\{eq:dual_zoom\}

Therefore, the essential homogeneity of $\mathbb{P} \in \mathbb{\Psi}_{H}^{m}(M)$ gives

$$
\beta_{\lambda}^{*} \hat{\mathbb{P}}-\lambda^{m} \hat{\mathbb{P}} \in \mathcal{S}_{r^{\prime}}\left(\mathfrak{t}_{H}^{*} M \times \mathbb{R}\right) \quad \text { for all } \lambda \in \mathbb{R}_{+}^{\times} .
$$


Importantly, the maps $\beta_{\lambda}$ are themselves dilations associated to a graded vector bundle. Namely, we change our point of view to consider $\mathfrak{t}_{H}^{*} M \times \mathbb{R}$ as a vector bundle over $M$ (rather than $M \times \mathbb{R}$ ) via the bundle projection

$$
\rho: \mathfrak{t}_{H}^{*} M \times \mathbb{R} \rightarrow M ; \quad(x, \eta, t) \mapsto x .
$$

\{eq: augmented_bundl

Then $\left(\beta_{\lambda}\right)$ is the family of dilations on this bundle, where $\mathfrak{t}_{H}^{*} M$ has its original grading and the complementary bundle $M \times \mathbb{R}$ is given grading degree 1.

With just a little care on our choice of $\mathbb{P} \in \Downarrow_{H}^{m}(M)$, we can organize for the homogeneity condition (24) to work modulo $\mathcal{S}_{\rho}\left(\mathfrak{t}_{H}^{*} M \times\right.$ $\mathbb{R})$ instead of just $\mathcal{S}_{r^{\prime}}\left(\mathfrak{t}_{H}^{*} M \times \mathbb{R}\right)$. For this, we need a short digression.

Definition 38. An essentially homogeneous $r$-fibred distribution $\mathbb{P} \in$ $\mathbb{v}_{H}^{m}(M)$ is called homogeneous on the nose outside $[-1,1]$ if for all $\lambda>1$,

$$
\left.\operatorname{supp}\left(\alpha_{\lambda *} \mathbb{P}-\lambda^{m} \mathbb{P}\right) \subset \mathbb{T}_{H} M\right|_{[-1,1]} .
$$

Any $P \in \Psi_{H}^{m}(M)$ admits an extension $\mathbb{P} \in \mathbb{\Psi}_{H}^{m}(M)$ which is homogeneous on the nose outside $[-1,1]$. This is not profound, but is a little technical, so to avoid interrupting the exposition, we have placed the proof in an appendix (Proposition 63). Note that exponential cut-off functions are also homogeneous on the nose, so we may simultaneously arrange that $\mathbb{P}$ be supported in a global exponential coordinate patch.

Returning now to the homogeneity of $\hat{\mathbb{P}}$, let $\mathbb{P} \in \mathbb{\Psi}_{H}^{m}(M)$ be homogeneous on the nose outside $[-1,1]$. Then the differences of Equation (24) have support bounded in $t$. We obtain the improved condition:

$$
\beta_{\lambda}^{*} \hat{\mathbb{P}}-\lambda^{m} \hat{\mathbb{P}} \in \mathcal{S}_{\rho}\left(\mathfrak{t}_{H}^{*} M \times \mathbb{R}\right) \quad \text { for all } \lambda \in \mathbb{R}_{+}^{\times} .
$$

Now, a bundle analogue of [Tay, Proposition 2.2] immediately gives the following.

Proposition 39. Let $\mathbb{P} \in \Downarrow_{H}^{m}(M)$ be homogeneous on the nose outside $[-1,1]$, and let $\hat{\mathbb{P}} \in C^{\infty}\left(\mathfrak{t}_{H}^{*} M \times \mathbb{R}\right)$ be its Fourier transform (with respect to any exponential coordinates). Then $\hat{\mathbb{P}}$ is equal, modulo $\mathcal{S}_{\rho}\left(\mathfrak{t}_{H}^{*} M \times \mathbb{R}\right)$, to a genuinely homogeneous function at infinity. More precisely, there exists a smooth function $A \in C^{\infty}\left(\mathfrak{t}_{H}^{*} M \backslash(M \times\{0\} \times\{0\})\right)$ satisfying

$$
\beta_{\lambda}^{*} A=\lambda^{m} A \quad \text { for all } \lambda \in \mathbb{R}_{+}^{\times},
$$

and such that, for any bump function $\varphi \in C^{\infty}\left(\mathfrak{t}_{H}^{*} M \times \mathbb{R}\right)$ which is 0 in some neighbourhood of the zero section of $\rho$ and 1 outside some $\rho$-proper set, we have $\hat{\mathbb{P}}-\varphi A \in \mathcal{S}_{\rho}\left(\mathfrak{t}_{H}^{*} M \times \mathbb{R}\right)$.

\{eq:homogeneity_of

\{prop: symbol_homoge 
Remark 40. Symbols of order $m$ are usually defined in terms of the symbol inequalities

$$
\left|\partial_{x}^{\mathbf{a}} \partial_{\eta}^{\mathbf{b}} a(x, \eta)\right| \leq C(1+\|\eta\|)^{m-|\mathbf{b}|}, \quad(x, \eta) \in T^{*} M .
$$

Note that these inequalities would all be satisfied automatically if $a \in C^{\infty}\left(T^{*} M\right)$ were a homogeneous function of order $m$ at infinity, perhaps modulo some Schwartz-class perturbation.

Of course, the full symbol $a \in C^{\infty}\left(T^{*} M\right)$ of a classical pseudodifferential operator is generally not homogeneous in this way-it is polyhomogeneous. The power of Proposition 39 is that the extension of $a=\hat{P}$ to $\hat{\mathbb{P}} \in C^{\infty}\left(T^{*} M \times \mathbb{R}\right)$ is homogeneous at infinity with respect to the augmented dilations $\left(\beta_{\lambda}\right)$. This $\beta$-homogeneity still immediately implies the symbol inequalities (25) upon restricting to $t=1$; see Corollary 41 below.

This simple observation explains the economy which is gained by passing to the tangent groupoid. For instance, in the case of the Heisenberg calculus, a great deal of the technical analysis of the book [BG88] is absorbed by this remark.

\section{Conormality AND Regularity}

With Proposition 39 in hand, we can now begin to prove the standard properties of pseudodifferential operators for our class $\Psi_{H}^{m}(M)$. This begins with decay estimates on the Fourier transform $\hat{\mathbb{P}}$.

8.1. Symbol estimates. Recall that a homogeneous norm on a graded vector space $V$ is a continuous function $\|\cdot\|_{H}: V \rightarrow[0, \infty)$ which is nonzero on $E \backslash\{0\}$ and which scales under the canonical dilations as

$$
\left\|\delta_{\lambda}(\xi)\right\|_{H}=\lambda\|\xi\|_{H}, \quad \xi \in V, \lambda \in \mathbb{R}_{+} .
$$

Corollary 41. Let $\mathbb{P} \in \Downarrow_{H}^{m}(M)$ be homogeneous on the nose outside $[-1,1]$. Fix a local exponential coordinate system $(x, \xi, t) \in M_{0} \times V \times$ $\mathbb{R} \hookrightarrow \mathfrak{t}_{H} M \times \mathbb{R}$ as in Section 5.3 and denote the dual coordinates by $(x, \eta, t) \in M_{0} \times V^{*} \times \mathbb{R} \hookrightarrow \mathfrak{t}_{H}^{*} M \times \mathbb{R}$. The Fourier transform $\hat{\mathbb{P}}$ in these coordinates satisfies the following estimates: For any compact $K \subset M_{0}$ and multi-indices $\mathbf{a}, \mathbf{b}, \mathbf{c} \in \mathbb{N}^{n}, k \in \mathbb{N}$, there is $C=C_{K, \mathbf{a}, \mathbf{b}, \mathbf{c}, k}>0$ such that for all $(x, \eta, t) \in K \times V^{*} \times \mathbb{R}$,

$$
\left|\eta^{\mathbf{a}} \partial_{\eta}^{\mathbf{b}} \partial_{x}^{\mathbf{c}} \partial_{t}^{k} \hat{\mathbb{P}}(x, \eta, t)\right| \leq C\left(1+\|\eta\|_{H}+|t|\right)^{m+|\mathbf{a}|_{H}-|\mathbf{b}|_{H}-k},
$$

where $\|\cdot\|_{H}$ is any homogeneous norm on $V^{*}$. 
Proof. From Proposition 39 the function $\eta^{\mathbf{a}} \partial_{\eta}^{\mathbf{b}} \partial_{x}^{\mathbf{c}} \partial_{t}^{k} \hat{\mathbb{P}}$ is equal, modulo a function in $\mathcal{S}_{\rho}\left(\mathfrak{t}_{H}^{*} M \times \mathbb{R}\right)$, to a function which is homogeneous at infinity of order $m+|\mathbf{a}|_{H}-|\mathbf{b}|_{H}-k$ with respect to $\left(\beta_{\lambda}\right)$.

We are now very close to the classical theory of pseudodifferential operators of type $\Psi_{\rho, \delta}^{m}$. To the expert, the results which follow will be no surprise.

8.2. Conormality. The first consequence of the estimates (26) is that our $H$-psuedodifferential kernels are proper distributions, i.e. are both $r$ - and $s$-fibered. For this, we follow a strategy of LescureManchon-Vassout [LMV15], showing that they have wavefront sets which are conormal to the diagonal. Here is the relevant lemma.

Lemma 42. Let $G$ be a Lie groupoid. Suppose $v \in \mathcal{D}^{\prime}(G)$ has proper support, singular support contained in $G^{(0)}$, and wave front set contained in the conormal to $G^{(0)}$ :

$$
\mathrm{WF}(v) \subseteq\left\{\left.(x, \eta) \in T^{*} G\right|_{G^{(0)}} \mid(\eta, \xi)=0 \text { for all } \xi \in T_{x} G^{(0)}\right\} .
$$

Then $v$ is a proper distribution.

Proof. Since $T\left(G^{(0)}\right)$ is complementary to the tangent space of the $r$-fibre at every point, the conormal bundle of $G^{(0)}$ intersects trivially with the conormal bundle of any $r$-fibre. Now apply [LMV15, Proposition 7] to conclude that $v$ can be disintegrated as an $r$-fibred distribution. Likewise, $v$ disintegrates as an $s$-fibred distribution.

We now prove that the conormality condition (27) holds for essentially homogeneous $r$-fibred distributions on $\mathbb{T}_{H} M$. Fix a transverse measure $\mu$ on the base of $\mathbb{T}_{H} M$, so that we have the integration maps $\mu_{r}: \mathcal{E}_{r}^{\prime}\left(\mathbb{T}_{H} M\right) \rightarrow \mathcal{D}^{\prime}\left(\mathbb{T}_{H} M\right)$ and $\mu_{s}: \mathcal{E}_{s}^{\prime}\left(\mathbb{T}_{H} M\right) \rightarrow \mathcal{D}^{\prime}\left(\mathbb{T}_{H} M\right)$ as in Section 2.5.

Proposition 43. Let $\mathbb{P} \in \Downarrow_{H}^{m}(M)$. The wave front set of the distribution $\mu_{r}(\mathbb{P}) \in \mathcal{D}^{\prime}\left(\mathbb{T}_{H} M\right)$ is conormal to the object space, i.e.,

$$
\begin{array}{r}
\mathrm{WF}\left(\mu_{r}(\mathbb{P})\right) \subseteq\left\{(x, \eta, t) \in T_{(x, t)}^{*}\left(\mathbb{T}_{H} M\right) \mid(x, t) \in M \times \mathbb{R}=\mathbb{T}_{H} M^{(0)}\right. \\
\text { and } \left.(\eta, \xi)=0 \text { for all } \xi \in T_{(x, t)}\left(\mathbb{T}_{H} M^{(0)}\right)\right\} .
\end{array}
$$

Thus $\mathbb{P}$ is a proper r-fibred distribution.

Proof of Proposition 43. We work in local exponential coordinates $M_{0} \times$ $V \times \mathbb{R} \rightarrow \mathbb{T}_{H} M$. It suffices to prove the proposition for $\mathbb{P}$ homogeneous on the nose outside $[-1,1]$, so we have the decay estimates (26). \{sec: conormality\}

\{decomposability\}

\{eq: conormality\}

\{prop: conormality\} 
Let us determine the wave front set of $\mu_{r}(\mathbb{P})$ at a point $\left(x_{0}, t_{0}\right) \in$ $M \times \mathbb{R}$. We may replace $\mathbb{P}$ with $\chi \mathbb{P}$, where $\chi \in C^{\infty}(M \times \mathbb{R})$ is some smooth bump function on the base which is 1 near $\left(x_{0}, t_{0}\right)$ and supported in some compact set $K \times I \subset M_{0} \times \mathbb{R}$. Since the fibrewise Fourier transform is $C^{\infty}(M \times \mathbb{R})$-linear, we still have decay conditions on $\widehat{\chi \mathbb{P}}$, in particular,

$$
\left|\partial_{x}^{c} \partial_{t}^{k}(\widehat{\chi \mathbb{P}})(x, \eta, t)\right| \leq C_{\mathbf{c}, k}\left(1+\|\eta\|_{H}\right)^{m}
$$

for every $\mathbf{c} \in \mathbb{N}^{n}, k \in \mathbb{N}$. The factor of $|t|$ has been removed from the right hand side, since the support of $\chi \mathbb{P}$ is bounded in $t$.

Now we apply the Fourier transform $\mathcal{F}_{(x, t) \rightarrow(y, s)}$ in the base variables. We obtain bounds

$$
\left|y^{\mathbf{c}} s^{k} \mathcal{F}_{(x, \xi, t) \rightarrow(y, \eta, s)}(\chi \widetilde{\mathbb{P}})(y, \eta, s)\right| \leq C_{\mathbf{c}, k} \mu(K)\left(1+\|\eta\|_{V^{*}}\right)^{m}
$$

for every $\mathbf{c} \in \mathbb{N}^{n}, k \in \mathbb{N}$. It follows that every $(y, \eta, s) \in \mathbb{R}^{n} \times \mathbb{R}^{n} \times \mathbb{R}$ with $(y, s) \neq(0,0)$ admits a conical neighbourhood upon which $\mathcal{F}_{(x, \xi, t) \rightarrow(y, \eta, s)}(\chi \widetilde{\mathbb{P}})$ has rapid decay. This proves the conormality of the wave front set.

Corollary 44. Elements of $\rrbracket_{H}^{m}(M)$ are proper r-fibred distributions. Thus, $H$-pseudodifferential kernels $P \in \Psi_{H}^{m}(M)$ are semiregular in both variables (see Proposition 13).

8.3. Algebra structure. Properness is needed to define the algebra structure on $\Psi_{H}^{\bullet}(M)$, since we need to know that $C_{\mathrm{p}}^{\infty}\left(\mathbb{T}_{H} M ; \Omega_{r}\right)$ is a two-sided ideal. This, plus the fact that $\alpha_{\lambda *}$ is an algebra automorphism of $\mathcal{E}_{r, s}^{\prime}\left(\mathbb{T}_{H} M\right)$ immediately yields the following.

Theorem 45. For any $m, m^{\prime} \in \mathbb{R}$ we have $\Downarrow_{H}^{m}(M) * \Downarrow_{H}^{m^{\prime}}(M) \subseteq \Downarrow_{H}^{m+m^{\prime}}(M)$. $\{$ thm:algebra\} Thus $\Psi_{H}^{\bullet}(M)=\bigcup_{m \in \mathbb{Z}} \Psi_{H}^{m}(M)$ is a filtered algebra.

Proposition 46. If $P \in \Psi_{H}^{m}(M)$ and $Q \in \Psi_{H}^{m^{\prime}}(M)$ then $\sigma_{m+m^{\prime}}(P * Q)=$ \{prop:symbol_is_alg $\sigma_{m}(P) * \sigma_{m^{\prime}}(Q)$.

Proof. If $\mathbb{P} \in \Psi_{H}^{m}$ and $\mathbb{Q} \in \Downarrow_{H}^{m^{\prime}}$ extend $P$ and $Q$, respectively, then $\mathbb{P} * \mathbb{Q} \in \mathbb{\Downarrow}_{H}^{m+m^{\prime}}$ extends $P * Q$. The result follows.

8.4. Regularity. Another consequence of the decay conditions (26) is the regularity of $H$-pseudodifferential kernels of large negative order.

Lemma 47. Let $\mathbb{P} \in \Downarrow_{H}^{-d_{H}-k-1}(M)$ for some $k \geq 0$, and $\widetilde{\mathbb{P}}$ be its pullback \{lem:regularity\} under some exponential coordinates $(x, \xi, t) \in M_{0} \times V \times \mathbb{R} \rightarrow \mathbb{T}_{H} M$. For any $\mathbf{a}, \mathbf{b} \in \mathbb{N}^{n}, c \in \mathbb{N}$ with $|\mathbf{a}|_{H} \leq k$, we have

$$
\partial_{\tilde{\xi}}^{\mathbf{a}} \partial_{x}^{\mathbf{b}} \partial_{t}^{c} \widetilde{\mathbb{P}} \in C\left(M_{0} \times V \times \mathbb{R} ; \Omega_{s}\right) .
$$


Proof. We assume $\mathbb{P}$ is homogeneous on the nose outside $[-1,1]$, so we have the decay estimates (26). For any multi-indices $\mathbf{a}, \mathbf{b} \in \mathbb{N}^{n}$, $c \in \mathbb{N}$ with $|\mathbf{a}|_{H} \leq k$, and for each $(x, t) \in M_{0} \times \mathbb{R}$, the function $\eta \mapsto$ $\eta^{\mathbf{a}} \partial_{x}^{\mathbf{b}} \partial_{t}^{c} \hat{\mathbb{P}}(x, \eta, t)$ is bounded in absolute value by $C^{\prime}\left(1+\|\eta\|_{H}\right)^{-d_{H}-1}$, which is integrable (see [NS79]). Taking fibrewise Fourier transforms gives $\partial_{\tilde{\xi}}^{\mathbf{a}} \partial_{x}^{\mathbf{b}} \partial_{t}^{c} \widetilde{\mathbb{P}} \in C\left(M_{0} \times V \times \mathbb{R} ; \Omega_{r}\right)$.

Theorem 48. Let $M$ be a smooth manifold equipped with a Lie filtration of depth $N$ and homogeneous dimension $d_{H}$. For any $k \geq 0$ we have $\rrbracket_{H}^{-d_{H}-k N-1}(M) \subseteq C^{k}\left(\mathbb{T}_{H} M ; \Omega_{r}\right)$ and $\Psi_{H}^{-d_{H}-k N-1}(M) \subseteq C^{k}(M \times$ $\left.M ; \Omega_{r}\right)$.

Proof. Follows from Lemma 47 since $|\mathbf{a}|_{H} \leq N|\mathbf{a}|$.

Corollary 49. If we put $\Psi_{H}^{-\infty}(M)=\bigcap_{m \in \mathbb{Z}} \Psi_{H}^{m}(M)$, then $\Psi_{H}^{-\infty}(M)=$ $\Psi^{-\infty}(M)=C_{\mathrm{p}}^{\infty}(M \times M)$ is the algebra of properly supported smoothing operators on $M$.

\section{AsYmptotic EXPANSIONS}

To construct parametrices, we need convergence of asymptotic expansions. We work with kernels, not symbols, meaning that the relevant notion of asymptotic convergence is as follows.

Definition 50. An aymptotic series of $H$-pseudodifferential operators will mean a series of the form $P_{0}+P_{1}+P_{2}+\cdots$ where $P_{k} \in \Psi_{H}^{m_{k}}(M)$ and the orders $m_{k}$ decrease to $-\infty$. We will say $P \in \Psi_{H}^{\bullet}(M)$ is an asymptotic limit of this series, denoted

$$
P \sim P_{0}+P_{1}+P_{2}+\cdots,
$$

if $P-\left(\sum_{k=1}^{j-1} P_{k}\right) \in \Psi_{H}^{m_{j}}(M)$ for all $j \in \mathbb{N}$.

Theorem 51. Every asymptotic series admits an asymptotic limit.

Proof. The proof is fairly standard: we modify the kernels $P_{k}$ successively using cut-off functions supported near the diagonal in order to make a genuinely convergent series. By collecting terms and dropping a finite number of them, it suffices to work with $P_{k} \in \Psi_{H}^{-d_{H}-k N-1}(M)$.

For each $k$, choose some $\mathbb{Q}_{k} \in \Downarrow_{H}^{-d_{H}-k N-1}(M)$ which extends $P_{k}$ and is homogeneous on the nose outside $[-1,1]$. Then $\mathbb{Q}_{k} \in$ $C^{k}\left(\mathbb{T}_{H} M ; \Omega_{r}\right)$ by Theorem 48 . After modifying $\mathbb{Q}_{k}$ (and thus $P_{k}$ ) by an element of $C_{\mathrm{p}}^{\infty}\left(\mathbb{T}_{H} M ; \Omega_{r}\right)$, we may assume without loss of generality that $\mathbb{Q}_{k}$ vanishes to order $k$ on $\mathbb{T}_{H} M^{(0)}$. Define $\mathbb{P}_{k}=t^{k N} \mathbb{Q}_{k} \in$ $\Psi_{H}^{-d_{H}-1}(M)$, which extends $P_{k}$, but as an element of order $-d_{H}-1$. Again, $\mathbb{P}-k$ vanishes to order $k$ on $\mathbb{T}_{H} M^{(0)}$.
$\{$ thm:k-regularity\}

\{cor:smoothing\}

$\{$ sec : asymp_exp\}

\{thm: completeness\} 
As usual, we write $\widetilde{\mathbb{P}}_{k} \in C^{k}\left(M_{0} \times V \times \mathbb{R} ; \Omega_{r}\right)$ for the pullback of $\mathbb{P}_{k}$ via some local exponential coordinates. Using a partition of unity on $M$, it is no loss of generality to suppose that $\widetilde{\mathbb{P}}_{k}$ is supported in $K \times V \times \mathbb{R}$ for some compact $K \subset M_{0}$.

We use the Lebesgue measure $\omega$ on $V$ to write $\mathbb{P}_{k}=p_{k} \omega$ with $p_{k} \in C^{k}\left(M_{0} \times V \times \mathbb{R}\right)$. An application of the Taylor Remainder Formula in $\xi$ shows that for all $\mathbf{a}, \mathbf{b} \in \mathbb{N}^{n}, c \in \mathbb{N}$ with $|\mathbf{a}| \leq k-1$ there is a constant $C_{K, \mathbf{a}, \mathbf{b}, c}>0$ such that

$$
\begin{aligned}
& \left|\partial_{\xi}^{\mathbf{a}} \partial_{x}^{\mathbf{b}} \partial_{t}^{c} p_{k}(x, \xi, t)\right| \leq C_{K, \mathbf{a}, \mathbf{b}, c}\|\xi\|^{k-|\mathbf{a}|} \\
& \qquad \text { for all }(x, \xi, t) \in K \times V \times[1,-1],
\end{aligned}
$$

where $\|\xi\|$ denotes the Euclidean norm of $\xi \in V$.

Now we proceed to cut off $p_{k}$ near $K \times\{0\} \times \mathbb{R}$. Fix a smooth bump function $\theta \in C^{\infty}(V)$ which is 1 on some neighbourhood of the origin and 0 outside the unit ball $\{\xi \in V \mid\|\xi\| \leq 1\}$. For $r \in \mathbb{R}_{+}^{\times}$, we define $\theta_{r}(\xi)=\theta\left(r^{-1} \xi\right)$, so that $\theta_{r}$ is supported in the ball of radius $r$ and its derivatives satisfy

$$
\left\|\partial_{\tilde{\xi}}^{\mathbf{a}} \theta_{r}\right\|_{\infty}=r^{-|\mathbf{a}|}\left\|\partial_{\tilde{\zeta}}^{\mathbf{a}} \theta\right\|_{\infty}
$$

for any multi-index $\mathbf{a} \in \mathbb{N}^{n}$. For each $k \geq 1$, we put

$$
r_{k}=\min \left(2^{-2 k}\left(\max _{|\mathbf{a}| \leq k-1}\left\|\partial_{\xi}^{\mathbf{a}} \theta\right\|_{\infty}\right)^{-1}\left(\max _{|\mathbf{a}|,|\mathbf{b}|, c \leq k-1} C_{K, \mathbf{a}, \mathbf{b}, c}\right)^{-1}, 1\right) .
$$

Define $\widetilde{\mathbb{P}}_{k}^{\prime}=p_{k}^{\prime} \omega$ with

$$
p_{k}^{\prime}(x, \xi, t)=\theta_{r_{k}}(\xi) p_{k}(x, \xi, t) .
$$

Then for all $|\mathbf{a}|,|\mathbf{b}|, c \leq k-1$ and all $(x, \xi, t) \in K \times V \times[-1,1]$ we have

$$
\begin{aligned}
\left|\partial_{\xi}^{\mathbf{a}} \partial_{x}^{\mathbf{b}} \partial_{t}^{c} p_{k}^{\prime}(x, \xi, t)\right| & =\left|\sum_{\mathbf{a}^{\prime} \leq \mathbf{a}}\left(\begin{array}{c}
\mathbf{a} \\
\mathbf{a}^{\prime}
\end{array}\right)\left(\partial_{\xi}^{\mathbf{a}-\mathbf{a}^{\prime}} \theta_{r_{k}}\right)(\xi)\left(\partial_{\xi}^{\mathbf{a}^{\prime}} \partial_{x}^{\mathbf{b}} \partial_{t}^{c} p_{k}\right)(x, \xi, t)\right| \\
& \leq \sum_{\mathbf{a}^{\prime} \leq \mathbf{a}}\left(\begin{array}{c}
\mathbf{a} \\
\mathbf{a}^{\prime}
\end{array}\right) r_{k}^{-\left|\mathbf{a}-\mathbf{a}^{\prime}\right|}\left\|\partial_{\xi}^{\mathbf{a}-\mathbf{a}^{\prime}} \theta\right\|_{\infty} C_{K, \mathbf{a}^{\prime}, \mathbf{b}, c} r_{k}^{k-\left|\mathbf{a}^{\prime}\right|} \\
& \leq r_{k}^{k-|\mathbf{a}|} \sum_{\mathbf{a}^{\prime} \leq \mathbf{a}}\left(\begin{array}{c}
\mathbf{a} \\
\mathbf{a}^{\prime}
\end{array}\right)\left\|\partial_{\xi}^{\mathbf{a}-\mathbf{a}^{\prime}} \theta\right\|_{\infty} C_{K, \mathbf{a}^{\prime}, \mathbf{b}, c} \\
& \leq 2^{-k-1},
\end{aligned}
$$

where $\left(\begin{array}{c}\mathbf{a} \\ \mathbf{a}^{\prime}\end{array}\right)=\prod_{i}\left(\begin{array}{l}a_{i} \\ a_{i}^{\prime}\end{array}\right)$ is a multi-binomial coefficient and the last line uses the formula $\sum_{\mathbf{a}^{\prime} \leq \mathbf{a}}\left(\begin{array}{c}\mathbf{a} \\ \mathbf{a}^{\prime}\end{array}\right)=2^{|\mathbf{a}|}$.

Therefore, for every $\mathbf{a}, \mathbf{b} \in \mathbb{N}^{n}$ and $c \in \mathbb{N}$, the series $\sum_{k \geq|\mathbf{a}|} \partial_{x}^{\mathbf{b}} \partial_{t}^{c} p_{k}^{\prime}$ is uniformly convergent on $K \times V \times[-1,1]$. It follows that the series 
$\sum_{k \geq 0} p_{k}^{\prime}$ converges to a limit $p \in C^{\infty}\left(M_{0} \times[-1,1] ; C(V)\right)$. Since every $p_{k}^{\prime}$ is homogeneous on the nose outside $[-1,1]$, this convergence extends to all of $\mathcal{E}_{r}^{\prime}\left(M_{0} \times V \times \mathbb{R}\right)$. We obtain

$$
\sum_{k \geq 0} \widetilde{\mathbb{P}}_{k}^{\prime}=\widetilde{\mathbb{P}}=p \omega \in \mathcal{E}_{r}^{\prime}\left(M_{0} \times V \times \mathbb{R}\right) .
$$

We need to check that $\mathbb{P} \in \Psi_{H}^{-d_{H}-1}(H)$, i.e., that it is essentially homogeneous of order $-d_{H}-1$. Fix any $k_{0} \geq 0$, and write

$$
\begin{aligned}
\alpha_{\lambda *} \mathbb{P}-\lambda^{-d_{H}-1} \mathbb{P}= & \sum_{k=0}^{k_{0}-1}\left(\alpha_{\lambda *} \mathbb{P}_{k}^{\prime}-\lambda^{-d_{H}-1} \mathbb{P}_{k}^{\prime}\right) \\
& \quad+\alpha_{\lambda *}\left(\sum_{k \geq k_{0}} \mathbb{P}_{k}^{\prime}\right)-\lambda^{-d_{H}-1}\left(\sum_{k \geq k_{0}} \mathbb{P}_{k}^{\prime}\right) .
\end{aligned}
$$

$\{$ eq: cocycle_sum\}

The first term on the right, being a finite sum of cocycles, belongs to $C^{\infty}\left(\mathbb{T}_{H} M ; \Omega_{r}\right)$. Meanwhile, $\sum_{k \geq k_{0}} \mathbb{P}_{k}^{\prime}$ is convergent in $C^{k_{0}}\left(\mathbb{T}_{H} M ; \Omega_{r}\right)$ by the preceding calculations. We deduce that $\alpha_{\lambda *} \mathbb{P}-\lambda^{-d_{H}-1} \mathbb{P}$ belongs to $C^{k_{0}}\left(\mathbb{T}_{H} M ; \Omega_{r}\right)$ for every $k_{0} \geq 0$, whence $\mathbb{P} \in \Psi_{H}^{-d_{H}^{-1}}(M)$.

Note that $\widetilde{\mathbb{P}}-\sum_{k=1}^{j-1} \widetilde{\mathbb{P}}_{k}^{\prime}=\sum_{k=j}^{\infty} \widetilde{\mathbb{P}}_{k}^{\prime}$ vanishes to order $j N-1$ at $t=0$, so by Proposition $34, \mathbb{P} \in \Downarrow_{H}^{-d_{h}-j N-1}(M)$. Therefore $P \sim \sum_{k} P_{k}$.

Remark 52. A remark on non-integer orders: given the definition of $H$-pseudodifferential operator, there is no inclusion $\Psi_{H}^{m}(M) \subset$ $\Psi_{H}^{m^{\prime}}(M)$ when $m^{\prime}-m \notin \mathbb{Z}$, since fractional powers of $t$ are not smooth. If one wishes to have an analogue of Theorem 51 for noninteger orders, one could define $\Phi_{H}^{\bullet+r}(M)=\bigcup_{m \in \mathbb{Z}} \Phi^{m+r}(M)$ for any fixed $r \in \mathbb{R}$. With the obvious definitions, Theorem 51 goes through essentially without change for asymptotic expansions in $\Phi_{H}^{\bullet+r}(M)$.

\section{H-ELLIPTICITY}

The convolution algebra $\mathcal{E}_{r}^{\prime}(G)$ on any Lie groupoid $G$ has an identity element $I$, namely the evaluation at the units:

$$
\langle I, f\rangle(x):=f(x), \quad f \in C^{\infty}(G), x \in G^{(0)} .
$$

We will denote the identity elements of $\mathcal{E}_{r}^{\prime}\left(\mathbb{T}_{H} M\right), \mathcal{E}_{r}^{\prime}(M \times M)$ and $\mathcal{E}_{r}^{\prime}\left(T_{H} M\right)$ by $\mathbb{I}, \mathbb{I}_{1}=I$ and $\mathbb{I}_{0}$, respectively.

Definition 53. An $H$-pseudodifferential operator $P \in \Psi_{H}^{m}(M)$ will $\{$ def:H-elliptic\} be called $H$-elliptic if its principal cosymbol $\sigma_{m}(P)$ admits a convolution inverse in $\mathcal{E}_{s}^{\prime}\left(T_{H} M\right) / C_{\mathrm{p}}^{\infty}\left(T_{H} M ; \Omega_{r}\right)$.

Lemma 54. The convolution inverse of a principal cosymbol of order $m$ (if it exists) is a principal cosymbol of order $-m$. 
Proof. Let $u \in \mathcal{E}_{r}^{\prime}\left(T_{H} M\right)$ represent a principal cosymbol of order $m$ with convolution inverse $v \in \mathcal{E}_{r}^{\prime}\left(T_{H} M\right)$ modulo $C_{\mathrm{p}}^{\infty}\left(T_{H} M ; \Omega_{r}\right)$. Working modulo $C_{\mathrm{p}}^{\infty}\left(T_{H} M ; \Omega_{r}\right)$, we have

$$
\begin{aligned}
& \delta_{\lambda *} v \equiv\left(\delta_{\lambda *} v\right) * u * v \equiv\left(\delta_{\lambda *} v\right) *\left(\lambda^{-m} \delta_{\lambda *} u\right) * v \\
& \equiv \lambda^{-m} \delta_{\lambda *}(I) * v=\lambda^{-m} v,
\end{aligned}
$$

for all $\lambda \in \mathbb{R}_{+}^{\times}$. The result follows.

Lemma 55. Let $P \in \Psi_{H}^{m}(M)$ be H-elliptic. Then there is $Q \in \Psi_{H}^{-m}(M)$ such that $P * Q-I$ and $Q * P-I$ are in $\Psi_{H}^{-1}(M)$.

Proof. Fix $\mathbb{P} \in \mathbb{\Psi}_{H}^{m}$ extending $P$. From the previous lemma and the surjectivity of the cosymbol map (Lemma 33) we can find $\mathbb{Q} \in \Downarrow_{H}^{-m}$ such that $\mathbb{P}_{0} * \mathbb{Q}_{0} \equiv \mathbb{I}_{0} \equiv \mathbb{Q}_{0} * \mathbb{P}_{0}$ modulo $C_{\mathrm{p}}^{\infty}\left(T_{H} M ; \Omega_{r}\right)$. Put $Q=$ $\mathbb{Q}_{1}$. The cosymbols $\sigma_{0}(P * Q-I)$ and $\sigma_{0}(Q * P-I)$ vanish, and the result follows from Lemma 35.

Definition 56. Let $P \in \Psi_{H}^{m}(M)$. An $H$-pseudodifferential operator $Q \in \Psi_{H}^{-m}(M)$ is called a parametrix for $P$ if $P * Q-I \in \Psi^{-\infty}(M)$ and $Q * P-I \in \Psi^{-\infty}(M)$.

Theorem 57. Every H-elliptic pseudodifferential operator $P \in \Psi_{H}^{m}(M)$ admits a parametrix.

Proof. This is a standard argument using asymptotic expansions. Using Lemma 55, pick $Q \in \Psi_{H}^{-m}(M)$ such that $R:=I-P * Q$ and $S:=I-Q * P$ are both in $\Psi_{H}^{-1}(M)$. Let us write $R^{k}$ for the $k$ th convolution power of $R$. The series $\sum_{k=0}^{\infty} R^{k}$ admits an asymptotic limit $A \in \Psi_{H}^{0}(M)$ by Theorem 51 .

Put $Q^{\prime}=Q * A$. Let $l \in \mathbb{N}$ be arbitrary. Working modulo $\Psi_{H}^{-(l+1)}(M)$ we have

$$
P * Q^{\prime}=P * Q * A \equiv(I-R) * \sum_{k=0}^{l} R^{k}=I-R^{l+1} \equiv I .
$$

Thus $P * Q^{\prime}-I \in \Psi^{-\infty}(M)$.

$$
\text { Also, } Q * R=S * Q \text {, so modulo } \Psi_{H}^{-(l+1)}(M) \text { we have }
$$

$$
\begin{aligned}
Q^{\prime} * P=Q * A * P \equiv\left(\sum_{k=0}^{l} S^{k}\right) * Q * P & \\
& =\left(\sum_{k=0}^{l} S^{k}\right) *(I-S)=I-S^{l+1} \equiv I .
\end{aligned}
$$

Hence $Q^{\prime} * P-I \in \Psi^{\infty}(M)$.

Corollary 58. Every H-elliptic differential operator is hypoelliptic. 


\section{Differential Operators}

$\{$ sec:diff_ops\}

In this section we spell out the details for the differential operators. To avoid technicalities, we shall assume that $M$ is compact ${ }^{2}$.

11.1. The universal enveloping algebra of a Lie algebroid. Let $G$ be a Lie group. It is well known that there are algebra isomorphisms between $(a)$ the set of left-invariant differential operators on $G,(b)$ the universal envelopping algebra $\mathcal{U}(\mathfrak{g})$, and $(c)$ the set of distributions on $G$ supported at the identity (an algebra under convolution). All of these have analogues for Lie groupoids.

The analogue of $(a)$ is the algebra $\mathrm{DO}_{r}(G)^{G}$ of (left-)invariant differential operators on $G$ which are tangent to the $r$-fibres. For $(b)$, one takes the universal enveloping algebra $\mathcal{U}(\mathfrak{A} G)$ of the Lie algebroid $\mathfrak{A G}$. For a definition, see e.g. ${ }^{3}$ [NWX99, Section 3]. For (c) we make the following definition.

Definition 59. We write $\mathcal{E}_{r}^{\prime}(G)^{(0)}$ for the set of $r$-fibred distributions on $G$ which are supported on the units space $G^{(0)}$.

Theorem 60. There are canonical algebra isomorphisms

$$
\mathcal{U}(\mathfrak{A G}) \cong \mathrm{DO}_{r}(G)^{G} \cong \mathcal{E}_{r}^{\prime}(G)^{(0)} .
$$

Specifically, the left-hand isomorphism is the unique extension of the map $\Gamma(\mathfrak{A} G) \rightarrow \Gamma\left(T_{r} M\right)$ induced by left-translation:

$$
i_{1}: X \mapsto \tilde{X}, \quad \text { where } \tilde{X}_{\gamma}:=L_{\gamma *} X_{s(\gamma)} .
$$

The right-hand isomorphism is $D \mapsto I \circ D$ where $I: C^{\infty}(G) \rightarrow C^{\infty}\left(G^{(0)}\right)$ is evaluation on the units. The inverse map is $u \mapsto D_{u}$ where

$$
D_{u} \varphi(\gamma):=\left\langle u^{s(\gamma)}, \varphi(\gamma \cdot)\right\rangle \text {. }
$$

11.2. Principal symbols. In the case of the pair groupoid, there is also an algebra isomorphism

$$
\mathrm{DO}_{r}(M \times M)^{M \times M} \cong \mathrm{DO}(M),
$$

where $\mathrm{DO}(M)$ designates the algebra of all finite order differential operators on $M$. Specifically, a differential operator $D \in \mathrm{DO}(M)$ is

\footnotetext{
${ }^{2}$ The specific technicality we are avoiding is that the order of a differential operator on a noncompact manifold may increase to infinity as we stray outward.

${ }^{3}$ Nistor-Weinstein-Xu [NWX99] use the Lie algebroid of tangent vectors to the source fibres, and invariant differential operators on the source fibres. The two pictures are intertwined by (the derivative of) the inverse map.

Note that if the unit space of $G$ is noncompact then the enveloping algebra $\mathcal{U}(\mathfrak{A G})$ has two possible variants, with or without a global bound on the order. Theorem 60 holds for each of these variants with the appropriate qualifiers on the global orders of differential operators and distributions.
}

$\{e q: D u\}$

$\{$ sec:diff_op_symbol 
mapped to the differential operator $\tilde{D} \in \mathrm{DO}_{r}(M \times M)$ which acts as $D$ on the second factor. The resulting isomorphism $\mathrm{DO}(M) \cong$ $\mathcal{E}_{r}^{\prime}(M \times M)^{(0)}$ sends a differential operator to its Schwartz kernel.

If $M$ is a filtered manifold, then we have a filtration on the sections of the Lie algebroid $\Gamma(T M)$ which is compatible with the Lie bracket. This extends to a filtration on the universal enveloping algebra $\mathcal{U}(T M) \cong \mathrm{DO}(M)$. We refer to this as the $H$-order of a differential operator. In the case of a trivially filtered manifold, this is the usual order. We denote the space of differential operators of $H$-order $\leq m$ by $\mathrm{DO}_{H}^{m}(M)$.

By abstract nonsense, the associated graded of the universal enveloping algebra $\mathcal{U}(T M) \cong \mathrm{DO}(M)$ of $T M$ is canonically identified with the universal enveloping algebra $\mathcal{U}\left(\mathfrak{t}_{H} M\right)$ of the associated graded of TM. This means that the grading maps $\sigma_{m}: \Gamma\left(H^{m}\right) \rightarrow$ $\Gamma\left(\mathfrak{t}_{H}^{m} M\right) \subset \Gamma\left(\mathfrak{t}_{H} M\right)$ extend canonically to give maps

$$
\sigma_{m}: \mathrm{DO}_{H}^{m}(M) \rightarrow \mathcal{U}\left(\mathfrak{t}_{H} M\right) .
$$

for each $m \in \mathbb{N}$. This defines the principal part of a differential operator of $\mathrm{H}$-order $m$.

To cut through the abstraction, if $D=X_{1} \ldots X_{k}$ is a monomial of vector fields where $X_{i}$ has $H$-order $m_{i}$, then $D$ has $H$-order $m=$ $\sum_{i} m_{i}$. Its principal part is $\sigma_{m}(D)=\sigma_{m_{1}}\left(X_{1}\right) \ldots \sigma_{m_{k}}\left(X_{k}\right)$.

11.3. Differential operators as $H$-pseudodifferential operators. Let $\{$ sec:diff_ops_homog $D$ be a differential operator on $M$ of $H$-order $m$. Its Schwartz kernel is $P=I \circ D \in \mathcal{E}_{r}^{\prime}(M \times M)^{(0)}$. Its principal part $\sigma_{m}(D) \in \mathcal{U}\left(\mathfrak{t}_{H} M\right)$ can be identified, by Theorem 60 , with an element of $\mathcal{E}_{r}^{\prime}\left(T_{H} M\right)^{(0)}$ which we will denote by $\sigma_{m}(P)$, since we will shortly see it is the principal cosymbol of $P$.

We may therefore associate to $D$ an $r$-fibred distribution $\mathbb{P}$ in $\mathcal{E}_{r}^{\prime}\left(\mathbb{T}_{H} M\right)^{(0)}$ as follows:

$$
\mathbb{P}_{t}= \begin{cases}t^{m} P, & t \neq 0 \\ \sigma_{m}(P), & t=0 .\end{cases}
$$

\{eq: ssigma\}

For a proof that this is a smooth family, we refer to [vEY].

The restriction of $\mathbb{P}$ to $M \times M \times \mathbb{R}^{\times}$is homogeneous on the nose of weight $m$. By continuity, this extends to $t=0$. We have thus proven that $D$ is an $H$-pseudodifferential operator of order $m$ with principal cosymbol $\sigma_{m}(P)$. This proves one half of Proposition 1 from the Introduction, which we state now in the filtered case.

Proposition 61. A semiregular kernel $P \in \mathcal{E}_{r}^{\prime}(M \times M)$ is the Schwartz \{prop:H-DOs\} kernel of a differential operator of $H$-order $\leq m$ if and only if $P=\left.\mathbb{P}\right|_{t=1}$ for some $\mathbb{P} \in \mathcal{E}_{r}^{\prime}(\mathbb{T} M)$ which is homogeneous on the nose of weight $m$. 
Proof. Let $\mathbb{P} \in \mathcal{E}_{S}^{\prime}\left(\mathbb{T}_{H} M\right)$ be homogeneous of weight $m$. The support of $\mathbb{P}$ is invariant under the zoom action on $\mathbb{T}_{H} M$. Since the only proper orbits of the zoom action are those contained in the unit space, $\operatorname{supp}(\mathbb{P}) \subseteq \mathbb{T}_{H} M^{(0)}$. Thus $P=\mathbb{P}_{1}$ is the kernel of a differential operator $D$ on $M$.

It remains to show that $D$ has $H$-order $\leq m$. Restricting to a relatively compact chart $M_{0} \subseteq M$, we may assume $D$ has finite order, say $n$. Suppose $n>m$. Then the family $t^{n} P$ on $\left.\mathbb{T}_{H} M\right|_{\mathbb{R}^{\times}}$ extends smoothly to $\sigma_{n}(P)$ at $t=0$. But $t^{n} P=t^{n-m} \mathbb{P}$ for $t \neq 0$, so by continuity, $\sigma_{n}(P)=0$. Thus $D$ has $H$-order $n-1$. Repeating this we arrive at $H$-order $m$.

\section{Classical Pseudodifferential Operators}

We conclude with the proof that our $H$-pseudodifferential operators coincide with the classical pseudodifferential operators in the case of a trivially filtered manifold $M$ (Theorem 2 of the Introduction).

For the terminology of classical pseudodifferential operators, we follow [Hör85, §18.1]. By restricting to local exponential coordinates, we may assume that $M$ is an open domain in $\mathbb{R}^{n}$. We equip $T M=M \times \mathbb{R}^{n}$ with the standard connection $\nabla$.

To begin with, suppose $P$ is (the Schwartz kernel of) a classical pseudodifferential operator of order $m$ on $M$. As remarked in Example 37, its symbol (in the usual sense, [Hör85, §18.1]) is given by

$$
a=\hat{P} \in C^{\infty}\left(M \times \hat{\mathbb{R}}^{n}\right) .
$$

By the definition of classical pseudodifferential operator, $a$ admits an asymptotic symbol expansion

$$
a(x, \eta) \sim \sum_{j} a_{j}(x, \eta)
$$

where $a_{j}$ is homogeneous of order $m-j$ in $\eta$ outside of $\|\eta\| \leq 1$ (see Definition 18.1 .5 of [Hör85]).

The inverse Fourier transforms $\check{a}_{j}=\mathcal{F}_{r}^{-1} a_{j} \in \mathcal{S}_{r}^{\prime}(T M)$ satisfy

$$
\delta_{\lambda *} \check{a}_{j}-\lambda^{m-j} \check{a}_{j} \in \mathcal{S}_{r}\left(T M ; \Omega_{r}\right), \quad \text { for all } \lambda \in \mathbb{R}^{\times}
$$

and have singular support on the zero section $M \times\{0\}$ (see e.g. [Tay]). Let us define the constant family of distributions

$$
\widetilde{\mathbb{P}}_{j}(x, \xi, t)=\varphi_{1}(x, \xi) \check{a}_{j}(x, \xi),
$$

where $\varphi_{1} \in C_{\mathrm{p}}(T M)$ is some bump function supported in a domain of injectivity for $\operatorname{Exp}^{\nabla}$. Since $\widetilde{\mathbb{P}}_{j}$ is essentially homogeneous with respect to the zoom action $\tilde{\alpha}$ on $T M \times \mathbb{R}$, its push-forward $\mathbb{P}_{j}$ via 
exponential coordinates belongs to $\Downarrow_{H}^{m-j}(M)$. The series $\left.\sum_{j} \mathbb{P}_{j}\right|_{t=1}$ admits an asymptotic limit $Q \in \Psi_{H}^{m}(M)$ in the sense of Section 9 .

By the regularity of $H$-pseudodifferential kernels (Theorem 48) and the analogous result for classical pseudodifferential operators, we see that for $k \gg 0$ both $P$ and $Q$ differ from $\operatorname{Exp}_{*}^{\nabla}\left(\sum_{j=1}^{k} \breve{a}_{j}\right)$ by a kernel in $C^{k-m-n}\left(M \times M ; \Omega_{r}\right)$. Thus $P-Q \in \Psi^{\infty}(M)$. This shows that $P$ is an $H$-pseudodifferential operator.

Conversely, suppose $P \in \Psi_{H}^{m}(M)$ is an $H$-pseudodifferential operator. By Corollary $41, a:=\hat{P}$ is in the symbol class $S^{m}\left(M \times \hat{\mathbb{R}}^{n}\right)$. It remains to prove that $a$ admits a polyhomogeneous expansion $a \sim \sum_{j} a_{j}$.

Choose $\mathbb{P} \in \mathbb{\Psi}_{H}^{m}(M)$ with $P=\mathbb{P}_{1}$. Put $B_{0}=\hat{\mathbb{P}}$. Note that $\left.B_{0}\right|_{t=0}$ is homogeneous of weight $m$ modulo $\mathcal{S}_{r^{\prime}}\left(T^{*} M\right)$ for the dilations $\delta_{\lambda}^{\prime}$ on $T^{*} M$. It is therefore equal, modulo $\mathcal{S}_{r^{\prime}}\left(T^{*} M\right)$, to a function $a_{0} \in C^{\infty}\left(T^{*} M\right)$ which is exactly homogeneous of weight $m$ outside of $\|\eta\| \leq 1$ (see [Tay, Proposition 2.2]). If we define $A_{0} \in C^{\infty}\left(T^{*} M \times\right.$ $\mathbb{R})$ by $\left.A_{0}\right|_{t}=a_{0}$ for all $t$, then

$$
\beta_{\lambda}^{*} A_{0}-\lambda^{m} A_{0} \in \mathcal{S}_{r^{\prime}}\left(t^{*} M \times \mathbb{R}\right) \quad \text { for all } \lambda \in \mathbb{R}_{+}^{\times} .
$$

It follows that $B_{1}:=t^{-1}\left(B_{0}-A_{0}\right)$ extends smoothly at $t=0$ to a function on $T^{*} M \times \mathbb{R}$ satisfying

$$
\beta_{\lambda}^{*} B_{1}-\lambda^{m-1} B_{1} \in \mathcal{S}_{r^{\prime}}\left(t^{*} M \times \mathbb{R}\right) \quad \text { for all } \lambda \in \mathbb{R}_{+}^{\times} .
$$

Note that $\left.B_{1}\right|_{t=1}=a-a_{0}$. Reasoning as for Corollary 41, we get $a-a_{0} \in S^{m-1}\left(M \times \hat{\mathbb{R}}^{n}\right)$. Also, $\left.B_{1}\right|_{t=0}$ is equal, modulo $\mathcal{S}_{r^{\prime}}\left(T^{*} M\right)$, to a function $a_{1} \in C^{\infty}\left(T^{*} M\right)$ which is exactly homogeneous of weight $m-1$ outside of $\|\eta\| \leq 1$.

Repeating, we define $A_{1} \in C^{\infty}\left(T^{*} M \times \mathbb{R}\right)$ by $A_{1} \mid t=a_{1}$ for all $t$ and then $B_{2}:=t^{-1}\left(B_{1}-A_{1}\right)$. Restricting to $t=1$ gives $a-a_{0}-a_{1} \in$ $S^{m-2}\left(M \times \hat{\mathbb{R}}^{n}\right)$. Continuing in this fashion, we obtain an asymptotic symbol expansion $a \sim \sum_{j=0}^{m} a_{j}$.

We have now proven the following.

Theorem 62. Let $M$ be a smooth manifold without boundary, equipped with the trivial filtration: $H^{1}=T M$. The algebra $\Psi_{H}^{\bullet}(M)$ of $H$-pseudodifferential operators is precisely the algebra of (Schwartz kernels of) classical pseudodifferential operators on $M$.

\section{Appendix A.}

The purpose of the appendix is to prove the following assertion from Section 7.3. 
Proposition 63. Any H-pseudodifferential operator $P$ admits an exten\{prop:homogeneous_o sion to $\mathbb{P} \in \Psi_{H}^{m}(M)$ which is homogeneous on the nose outside $[-1,1]$.

This is not difficult, except for one technical point: we will need to know that the cocycle $F_{\lambda}$ associated to any $\mathbb{P} \in \Downarrow_{H}^{m}(M)$ is smooth as a function of $\lambda$. It is immediate from the definitions that $F$ is smooth as a function into distributions $\mathcal{E}^{\prime}\left(\mathbb{T}_{H} M\right)$, but the cocycle $F$ takes values in $C_{\mathrm{p}}^{\infty}\left(\mathbb{T}_{H} M ; \Omega_{r}\right)$, for which the appropriate topology is the LF-topology given by

$$
C_{\mathrm{p}}^{\infty}\left(\mathbb{T}_{H} M ; \Omega_{r}\right)=\underset{\lim }{\longrightarrow} C^{\infty}\left(X_{i} ; \Omega_{r}\right),
$$

where $\left(X_{i}\right)_{i \in \mathbb{N}}$ is a countable exhaustion of $\mathbb{T}_{H} M$ by proper subsets which are the closure of their interiors.

Lemma 64. For any $\mathbb{P} \in \Downarrow_{H}^{m}(M)$, the associated cocycle $F: \lambda \mapsto$ \{lem:smooth_cocycle $\lambda^{-m} \alpha_{\lambda *} \mathbb{P}-\mathbb{P}$ is a smooth function of $\mathbb{R}_{+}^{\times}$into $C_{\mathrm{p}}^{\infty}\left(\mathbb{T}_{H} M ; \Omega_{r}\right)$.

Proof. For $\lambda$ in any fixed closed interval $[a, b] \subset \mathbb{R}_{+}^{\times}$, the functions $F_{\lambda}$ are all supported in a common proper set $X:=\bigcup_{\lambda \in[a, b]} \alpha_{\lambda}(\operatorname{supp} \mathbb{P})$, so it will suffice to prove that $F$ is smooth as a function into $C^{\infty}\left(\mathbb{T}_{H} M ; \Omega_{r}\right)$.

To this end, we consider the restriction of $F_{\lambda}$ to the $r$-fibres over some compact domain $K \times I$ in the unit space $M \times \mathbb{R}$. Let us write $X_{0}:=X \cap r^{-1}(K \times I)$.

The key step is to prove the continuity of $F$, for which we use a Baire category argument. Fix $R>0$ and $k \in \mathbb{N}$. We put

$$
I_{k, R}=\left\{\lambda \in \mathbb{R}_{+}^{\times}\left|\left\|\left.F_{\lambda}\right|_{X_{0}}\right\|_{C^{k}} \leq R\right\} \subseteq \mathbb{R}_{+}^{\times} .\right.
$$

Consider some $\lambda \in \mathbb{R}_{+}^{\times}$in the closure of $I_{k, R}$. Let $\lambda_{i} \in I_{k, R}$ with $\lambda_{i} \rightarrow \lambda$. Then $F_{\lambda_{i}} \rightarrow F_{\lambda}$ in the $\mathcal{E}_{r}^{\prime}$-topology but also, by the ArzelaAscoli Theorem, some subsequence of $\left.F_{\lambda_{i}}\right|_{X_{0}}$ converges in the $C^{k-1}$ topology. Therefore $\left.F_{\lambda}\right|_{X_{0}} \in C^{k-1}\left(X_{0} ; \Omega_{r}\right)$. This proves that $\overline{I_{k, R}} \subseteq$ $I_{k-1, R}$. Moreover, $\left.\lambda \mapsto F_{\lambda}\right|_{X_{0}}$ is continuous from $\overline{I_{k, R}}$ to $C^{k-1}\left(X_{0} ; \Omega_{r}\right)$.

Since $\mathbb{R}_{+}^{\times}=\bigcup_{R \in \mathbb{N}} I_{k, R}$, the Baire Category Theorem shows there is some $R \in \mathbb{N}$ such that $\overline{I_{k, R}}$ contains an open interval $(a, b)$. Let $t \in \mathbb{R}_{+}^{\times}$be arbitrary and consider the function

$$
\begin{aligned}
\mathbb{R}_{+}^{\times} & \rightarrow C^{k-1}\left(X_{0} ; \Omega_{r}\right) \\
\lambda & \left.\mapsto F_{\lambda t}\right|_{X_{0}}=\left.\lambda^{-m} \alpha_{\lambda *} F_{t}\right|_{X_{0}}+\left.F_{\lambda}\right|_{X_{0}} .
\end{aligned}
$$

Note that $\lambda \mapsto \lambda^{-m} \alpha_{\lambda *} F_{t}$ is a smooth function of $\mathbb{R}_{+}^{\times}$into $C^{\infty}\left(\mathbb{T}_{H} M ; \Omega_{r}\right)$ so, by the previous paragraph, the map (34) is continuous for $\lambda \in$ $(a, b)$. In other words $\left.\lambda \mapsto F_{\lambda}\right|_{X_{0}}$ is continuous from $(t a, t b)$ to 
$C^{k-1}\left(X_{0} ; \Omega_{r}\right)$, and hence continuous on all of $\mathbb{R}_{+}^{\times}$since $t$ was aribtrary. This is true for any $k$, so $F: \mathbb{R}_{\times}^{+} \rightarrow C^{\infty}\left(\mathbb{T}_{H} M ; \Omega_{r}\right)$ is continuous.

Finally, to pass from continuity to smoothness will use a convolution trick. Pick a compactly supported bump function $\phi \in C_{\mathrm{c}}^{\infty}\left(\mathbb{R}_{+}^{\times}\right)$ with $\int_{\mathbb{R}_{+}^{\times}} \phi(t) \frac{d t}{t}=1$, and define $\mathbb{Q} \in \mathcal{E}^{\prime}\left(\mathbb{T}_{H} M ; \Omega_{r}\right)$ as an average of translates of $\mathbb{P}$ :

$$
\mathbb{Q}:=\int_{\mathbb{R}_{+}^{\times}} \phi(t) t^{-m}\left(\alpha_{t *} \mathbb{P}\right) \frac{d t}{t} \in \mathcal{E}_{s}^{\prime}\left(\mathbb{T}_{H} M\right) .
$$

Then $\mathbb{Q}-\mathbb{P}=\int_{\mathbb{R}_{+}^{\times}} \phi(t) F_{t} \frac{d t}{t}$, which belongs to $C^{\infty}\left(\mathbb{T}_{H} M ; \Omega_{s}\right)$ since the integrand is continuous and compactly supported. Thus $\lambda \mapsto$ $\lambda^{-m} \alpha_{\lambda *}(\mathbb{Q}-\mathbb{P})$ is a smooth function $\mathbb{R}_{+}^{\times} \rightarrow C^{\infty}\left(\mathbb{T}_{H} M ; \Omega_{S}\right)$.

Also,

$$
\begin{aligned}
\left(\lambda^{-m} \alpha_{\lambda *} \mathbb{Q}\right)-\mathbb{P} & =\int_{\mathbb{R}_{\times}^{+}} \phi(t)\left((\lambda t)^{-m} \alpha_{\lambda t *} \mathbb{P}-\mathbb{P}\right) \frac{d t}{t} \\
& =\int_{\mathbb{R}_{\times}^{+}} \phi\left(\lambda^{-1} t\right) F_{t} \frac{d t}{t}
\end{aligned}
$$

and by differentiation under the integral we see that this is a smooth function of $\lambda \in \mathbb{R}_{+}^{\times}$into $C^{\infty}\left(\mathbb{T}_{H} M ; \Omega_{S}\right)$. So, by writing

$$
F_{\lambda}=\lambda^{-m} \alpha_{\lambda *}(\mathbb{P}-\mathbb{Q})+\left(\lambda^{-m} \alpha_{\lambda *} \mathbb{Q}-\mathbb{P}\right),
$$

we obtain that $F$ is smooth.

Proof of Proposition 63. Let $P \in \Psi_{H}^{m}(M)$. Choose any $\mathbb{Q} \in \Downarrow_{H}^{m}(M)$ with $\mathbb{Q}_{1}=P$. Define $r$-fibred distributions $\mathbb{P}^{ \pm}$on $\left.\mathbb{T}_{H} M\right|_{\mathbb{R}_{ \pm}^{\times}}=M \times$ $M \times \mathbb{R}_{ \pm}^{\times}$by $\mathbb{P}_{t}^{ \pm}=|t|^{m} \mathbb{Q}_{ \pm 1}$. For all $t>0$ we have

$$
\mathbb{P}_{t}^{+}-\mathbb{Q}_{t}=\left(t^{m} \alpha_{t^{-1} *} \mathbb{Q}-\mathbb{Q}\right)_{t},
$$

which, by the previous Lemma, is a smooth function of $t$ valued in $C_{\mathrm{p}}^{\infty}\left(M \times M ; \Omega_{r}\right)$. That is, $\mathbb{P}^{+}-\mathbb{Q}_{\mathbb{R}_{+}^{\times}} \in C_{\mathrm{p}}^{\infty}\left(\left.\mathbb{T}_{H} M\right|_{\mathbb{R}_{+}^{\times}} ; \Omega_{r}\right)$. Likewise, $\mathbb{P}^{-}-\left.\mathbb{Q}\right|_{\mathbb{R}_{-}^{\times}} \in C_{\mathrm{p}}^{\infty}\left(\left.\mathbb{T}_{H} M\right|_{\mathbb{R}_{-}^{\times}} ; \Omega_{r}\right)$

Now let $\left\{\rho^{-}, \rho^{0}, \rho^{+}\right\}$be a smooth partition of unity on $\mathbb{R}$ with supports in $\left(-\infty,-\frac{1}{2}\right),(-1,1),\left(\frac{1}{2}, \infty\right)$, respectively and define $\mathbb{P}$ by

$$
\mathbb{P}_{t}=\rho^{-}(t) \mathbb{P}_{t}^{-}+\rho^{0}(t) \mathbb{Q}_{t}+\rho^{+}(t) \mathbb{P}_{t}^{+} \quad(t \in \mathbb{R}) .
$$

Then $\mathbb{P}-\mathbb{Q} \in C_{\mathrm{p}}^{\infty}\left(\mathbb{T}_{H} M ; \Omega_{r}\right)$, so $\mathbb{P} \in \Downarrow_{H}^{m}(M)$. It is homogeneous on the nose outside $[-1,1]$, and $\mathbb{P}_{1}=P$. 


\section{REFERENCES}

[AS11] Iakovos Androulidakis and Georges Skandalis. Pseudodifferential calculus on a singular foliation. J. Noncommut. Geom., 5(1):125-152, 2011.

[BG88] Richard Beals and Peter Greiner. Calculus on Heisenberg manifolds, volume 119 of Annals of Mathematics Studies. Princeton University Press, Princeton, NJ, 1988.

[CGGP92] Michael Christ, Daryl Geller, Paweł Głowacki, and Larry Polin. Pseudodifferential operators on groups with dilations. Duke Math. J., 68(1):31-65, 1992.

[Con94] Alain Connes. Noncommutative geometry. Academic Press, Inc., San Diego, CA, 1994.

[CP15] Woocheol Choi and Raphael Ponge. Privileged coordinates and tangent groupoid for carnot manifolds. Preprint. http://arxiv.org/abs/1510.05851. 2015.

[CR08] Paulo Carrillo Rouse. A Schwartz type algebra for the tangent groupoid. In K-theory and noncommutative geometry, EMS Ser. Congr. Rep., pages 181-199. Eur. Math. Soc., Zürich, 2008.

[ČS09] Andreas Čap and Jan Slovák. Parabolic geometries. I, volume 154 of Mathematical Surveys and Monographs. American Mathematical Society, Providence, RI, 2009. Background and general theory.

[Cum89] Thomas E. Cummins. A pseudodifferential calculus associated to 3step nilpotent groups. Comm. Partial Differential Equations, 14(1):129171, 1989.

[DS14] Claire Debord and Georges Skandalis. Adiabatic groupoid, crossed product by $\mathbb{R}_{+}^{*}$ and pseudodifferential calculus. Adv. Math., 257:66-91, 2014.

[EM] Charles Epstein and Richard Melrose. The heisenberg algebra, index theory and homology. Unpublished book, http://www-math.mit.edu/ rbm/book.html

[FS74] G. B. Folland and E. M. Stein. Estimates for the $\bar{\partial}_{b}$ complex and analysis on the Heisenberg group. Comm. Pure Appl. Math., 27:429-522, 1974.

[Hör85] Lars Hörmander. The analysis of linear partial differential operators. III, volume 274 of Grundlehren der Mathematischen Wissenschaften [Fundamental Principles of Mathematical Sciences]. Springer-Verlag, Berlin, 1985. Pseudodifferential operators.

[Jul02] Pierre Julg. La conjecture de Baum-Connes à coefficients pour le groupe Sp(n,1). C. R. Math. Acad. Sci. Paris, 334(7):533-538, 2002.

[JvE10] Pierre Julg and Erik van Erp. The geometry of the osculating nilpotent group structures of the heisenberg calculus. Preprint. http://arxiv.org/abs/1007.4759, 2010.

[LMV15] Jean-Marie Lescure, Dominique Manchon, and Stéphane Vassout. About the convolution of distributions on groupoids. Preprint. http://arxiv.org/abs/1502.02002, 2015.

[Mac87] K. Mackenzie. Lie groupoids and Lie algebroids in differential geometry, volume 124 of London Mathematical Society Lecture Note Series. Cambridge University Press, Cambridge, 1987.

[Mel82] Anders Melin. Lie filtrations and pseudo-differential operators. Preprint, 1982. 
[MM03] I. Moerdijk and J. Mrčun. Introduction to foliations and Lie groupoids, volume 91 of Cambridge Studies in Advanced Mathematics. Cambridge University Press, Cambridge, 2003.

[NS79] Alexander Nagel and E. M. Stein. Lectures on pseudodifferential operators: regularity theorems and applications to nonelliptic problems, volume 24 of Mathematical Notes. Princeton University Press, Princeton, N.J.; University of Tokyo Press, Tokyo, 1979.

[NWX99] Victor Nistor, Alan Weinstein, and Ping Xu. Pseudodifferential operators on differential groupoids. Pacific J. Math., 189(1):117-152, 1999.

[Pon06] Raphaël Ponge. The tangent groupoid of a Heisenberg manifold. Pacific J. Math., 227(1):151-175, 2006.

[Pon08] Raphaël S. Ponge. Heisenberg calculus and spectral theory of hypoelliptic operators on Heisenberg manifolds. Mem. Amer. Math. Soc., 194(906):viii+ 134, 2008.

[Tay] Michael Taylor. Noncommutative microlocal analysis, part I (revised version). http://www.unc.edu/math/Faculty/met/NCMLMS.pdf.

[Trè80] François Trèves. Introduction to pseudodifferential and Fourier integral operators. Vol. 1. Plenum Press, New York-London, 1980. Pseudodifferential operators, The University Series in Mathematics.

[vE05] Erik van Erp. The Atiyah-Singer index formula for subelliptic operators on contact manifolds. ProQuest LLC, Ann Arbor, MI, 2005. Thesis (Ph.D.)The Pennsylvania State University.

[vE10] Erik van Erp. The Atiyah-Singer index formula for subelliptic operators on contact manifolds. Part I. Ann. of Math. (2), 171(3):1647-1681, 2010.

[vEY] Erik van Erp and Robert Yuncken. On the tangent groupoid of a filtered manifold. In preparation.

Department of Mathematics, Dartmouth College, Hanover, NH 03755, USA

E-mail address: erikvanerp@dartmouth.edu

Université Clermont Auvergne, Université Blaise Pascal, BP 10448, F63000 Clermont-Ferrand, France

E-mail address: yuncken@math.univ-bpclermont.fr 